\title{
Ensemble Theory for Stealthy Hyperuniform Disordered Ground States
}

\author{
S. Torquato" \\ Department of Chemistry, Department of Physics, \\ Princeton Institute for the Science and Technology of Materials, \\ and Program in Applied and Computational Mathematics, Princeton University, \\ Princeton, New Jersey 08544, USA \\ G. Zhang and F. H. Stillinger \\ Department of Chemistry, Princeton University, Princeton, New Jersey 08544, USA \\ (Received 9 January 2015; published 29 May 2015)
}

\begin{abstract}
It has been shown numerically that systems of particles interacting with isotropic "stealthy" bounded long-ranged pair potentials (similar to Friedel oscillations) have classical ground states that are (counterintuitively) disordered, hyperuniform, and highly degenerate. Disordered hyperuniform systems have received attention recently because they are distinguishable exotic states of matter poised between a crystal and liquid that are endowed with novel thermodynamic and physical properties. The task of formulating an ensemble theory that yields analytical predictions for the structural characteristics and other properties of stealthy degenerate ground states in $d$-dimensional Euclidean space $\mathbb{R}^{d}$ is highly nontrivial because the dimensionality of the configuration space depends on the number density $\rho$ and there is a multitude of ways of sampling the ground-state manifold, each with its own probability measure for finding a particular ground-state configuration. The purpose of this paper is to take some initial steps in this direction. Specifically, we derive general exact relations for thermodynamic properties (energy, pressure, and isothermal compressibility) that apply to any ground-state ensemble as a function of $\rho$ in any $d$, and we show how disordered degenerate ground states arise as part of the ground-state manifold. We also derive exact integral conditions that both the pair correlation function $g_{2}(r)$ and structure factor $S(k)$ must obey for any $d$. We then specialize our results to the canonical ensemble (in the zero-temperature limit) by exploiting an ansatz that stealthy states behave remarkably like "pseudo"-equilibrium hard-sphere systems in Fourier space. Our theoretical predictions for $g_{2}(r)$ and $S(k)$ are in excellent agreement with computer simulations across the first three space dimensions. These results are used to obtain order metrics, local number variance, and nearest-neighbor functions across dimensions. We also derive accurate analytical formulas for the structure factor and thermal expansion coefficient for the excited states at sufficiently small temperatures for any $d$. The development of this theory provides new insights regarding our fundamental understanding of the nature and formation of low-temperature states of amorphous matter. Our work also offers challenges to experimentalists to synthesize stealthy ground states at the molecular level.
\end{abstract}

DOI: 10.1103/PhysRevX.5.021020

\section{INTRODUCTION}

The equilibrium structure and phase behavior of soft matter systems span from the relatively simple, as found in strongly repulsive colloidal particles, to the highly complex, as seen in microemulsions and polymers [1-10]. Soft matter has been fruitfully microscopically modeled as classical many-particle systems in which the particles (or metaparticles) interact with effective pair potentials. Bounded (soft) effective interactions have been particularly

\footnotetext{
*torquato@electron.princeton.edu
}

Published by the American Physical Society under the terms of the Creative Commons Attribution 3.0 License. Further distribution of this work must maintain attribution to the author(s) and the published article's title, journal citation, and DOI.
Subject Areas: Soft Matter, Statistical Physics

useful in modeling polymer systems, and they display zerotemperature ground states with a rich variety of crystalline structures, depending on the composition of the constituents and interaction parameters [1,4,7-10].

We have previously used a "collective-coordinate" approach to generate numerically exotic classical ground states of many particles interacting with certain bounded isotropic long-ranged pair potentials in one-, two-, and three-dimensional Euclidean space dimensions [11-17] as well as with anisotropic potentials [18]. It was shown that the constructed ground states across dimensions are the expected crystal structures in a low-density regime $[11,12,14,19]$, but above some critical density, there is a phase transition to ground states that are, counterintuitively, disordered (statistically isotropic with no long-range order), hyperuniform, and highly degenerate [20]. These unusual 
amorphous states of matter have been shown to be endowed with novel thermodynamic and physical properties [14-16,25-27] and belong to the more general class of disordered "hyperuniform" systems, which have been attracting attention recently, as detailed below.

The disordered ground states are highly degenerate with a configurational dimensionality that depends on the density, and there are an infinite number of distinct ways to sample this complex ground-state manifold, each with its own probability measure. For these reasons, it is theoretically very challenging to devise ensemble theories that are capable of predicting structural attributes and other properties of the ground-state configurations. A new type of statistical-mechanical theory must be invented to characterize these exotic states of matter. The purpose of this paper is to take some initial steps in this direction. However, to motivate the theoretical formalism, it is instructive to first briefly review the collective-coordinate numerical procedure that we have used to achieve disordered ground states.

In the simplest setting, we previously examined pairwise additive potentials $v(\mathbf{r})$ that are bounded and integrable such that their Fourier transforms $\tilde{v}(\mathbf{k})$ exist. If $N$ identical point particles reside in a fundamental region $F$ of volume $v_{F}$ in $d$-dimensional Euclidean space $\mathbb{R}^{d}$ at positions $\mathbf{r}^{N} \equiv$ $\mathbf{r}_{1}, \ldots, \mathbf{r}_{N}$ under periodic boundary conditions, the total potential energy can be expressed in terms of $\tilde{v}(\mathbf{k})$ as follows:

$$
\Phi\left(\mathbf{r}^{N}\right)=\frac{1}{2 v_{F}}\left[\sum_{\mathbf{k}} \tilde{v}(\mathbf{k})|\tilde{n}(\mathbf{k})|^{2}-N \sum_{\mathbf{k}} \tilde{v}(\mathbf{k})\right],
$$

where $\tilde{n}(\mathbf{k})=\sum_{j=1}^{N} \exp \left(-i \mathbf{k} \cdot \mathbf{r}_{j}\right)$ is the complex collective density variable, which can be viewed as a nonlinear transformation from the finite set of particle coordinates $\mathbf{r}_{1}, \ldots, \mathbf{r}_{N}$ to the complex functions $\tilde{n}(\mathbf{k})$ that depend on the infinite set of wave vectors $\mathbf{k}$ in reciprocal space appropriate to the fundamental cell $F$. The crucial idea is that if $\tilde{v}(\mathbf{k})$ is defined to be bounded and positive with support in the radial interval $0 \leq|\mathbf{k}| \leq K$ and if the particles are arranged so that $|\tilde{n}(\mathbf{k})|^{2}$, a quantity proportional to the structure factor $S(\mathbf{k})$, is driven to its minimum value of zero for all wave vectors where $\tilde{v}(\mathbf{k})$ has support (except $\mathbf{k}=\mathbf{0}$ ), then it is clear from relation (1) that the system must be at its ground state or global energy minimum. We have referred to these ground-state configurations as "stealthy" [14] because the structure factor $S(\mathbf{k})$ (scattering pattern) is zero for $0<|\mathbf{k}| \leq K$, meaning that they completely suppress single scattering of incident radiation for these wave vectors and, thus, are transparent at the corresponding wavelengths [28]. Various optimization techniques were employed to find the globally energyminimizing configurations within an exceedingly small numerical tolerance [11-18]. Generally, a numerically obtained ground-state configuration depends on the number of particles $N$ within the fundamental cell, initial particle configuration, shape of the fundamental cell, and particular optimization technique employed.

As the number of $\mathbf{k}$ vectors for which $|\tilde{n}(\mathbf{k})|$ is constrained to be zero increases, i.e., as $K$ increases, the dimensionality of the ground-state configuration manifold $D_{C}$ decreases. Because $|\tilde{n}(\mathbf{k})|$ is inversion symmetric, the number of wave vectors contained in a sphere of radius $K$ centered at the origin must be an odd integer, say $2 M(K)+1$, and thus $M(K)$ is the number of independently constrained wave vectors [29]. The parameter

$$
\chi=\frac{M(K)}{d(N-1)},
$$

which is inversely proportional to density, gives a measure of the relative fraction of constrained degrees of freedom compared to the total number of degrees of freedom $d(N-1)$ (subtracting out the system translational degrees of freedom). We show in Sec. IV B that the dimensionality of the configuration space per particle is given by $d(1-2 \chi)$ in the thermodynamic limit.

It is straightforward to see why, for sufficiently small $\chi$, ground states exist that are highly degenerate and typically disordered for sufficiently large $N$; see Fig. 1. Clearly, when the system is free of any constraints, i.e., if $\chi=0$, it is a noninteracting classical ideal gas. While it is unusual to think of classical ideal-gas configurations as ground states, at $T=0$, they indeed are global energy-minimizing states that are highly degenerate and typically disordered for large enough $N$. While the ground-state manifold contains periodic configurations (e.g., Bravais lattices and lattices with a basis), these are sets of zero measure in the thermodynamic limit. Clearly, if $\chi$ is made positive but very small, the ground states remain disordered and highly degenerate, even if the dimensionality of the configuration space $D_{C}$ has now been suddenly reduced due to the imposed constrained degrees of freedom, the number of which is determined by the radius $K$. From relation (2), we see that if $K$ is fixed, configurations with ideal-gas-like pair correlation functions correspond to the limit $\chi \rightarrow 0$ or, equivalently, to the limit $\rho \rightarrow \infty$ [30]. The latter situation runs counter to traditional understanding that ideal-gas configurations correspond to the opposite zero-density limit of classical systems of particles. The reason for this inversion of limits is due to the fact that a compression of the system in direct space leads to a dilation of the lattice spacing in reciprocal space, as schematically shown in Fig. 1. While it is not surprising that the configuration space is fully connected for sufficiently small $\chi$, quantifying its topology as a function of $\chi$ for all allowable $\chi$ is an outstanding problem, which is discussed further in the Conclusions.

It is noteworthy that stealthy point patterns (disordered or not) constitute a special class of so-called hyperuniform 


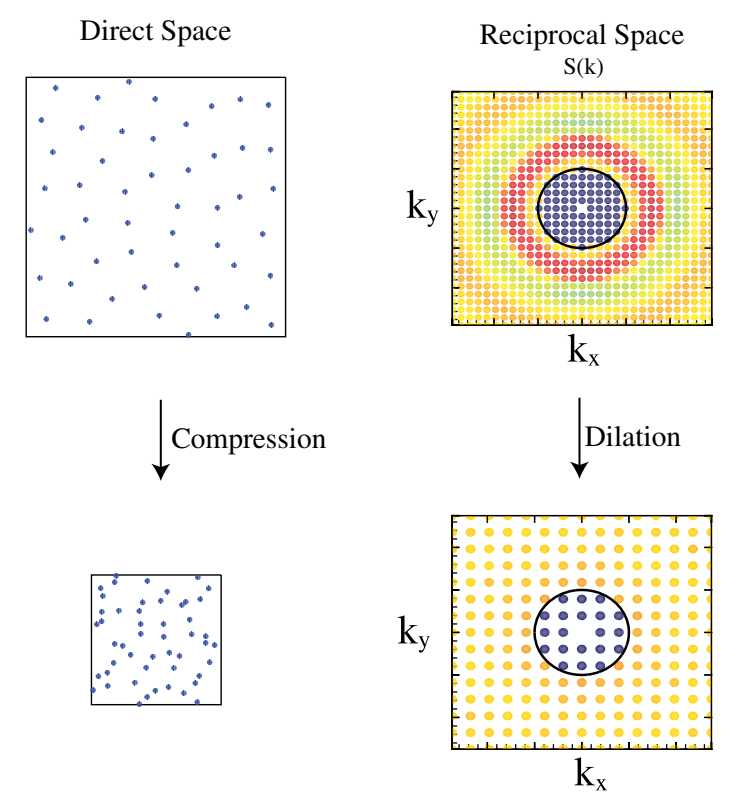

FIG. 1. Schematic illustrating the inverse relationship between the direct-space number density $\rho$ and relative fraction of constrained degrees of freedom $\chi$ for a fixed reciprocal-space exclusion-sphere radius $K$ (where dark blue $\mathbf{k}$ points signify zero intensity with green, yellow, and red points indicating increasingly larger intensities) for a stealthy ground state. A compression of a disordered ground-state configuration with a fixed number of particles $N$ in direct space leads to a dilation of the lattice spacing in reciprocal space. This means that during the compression process, the $\mathbf{k}$ points for which $|\tilde{n}(\mathbf{k})|$ is zero associated with the initial uncompressed system move out of the exclusion zone; i.e., the value of $M(K)$ [cf. Eq. (2)] decreases. Since there are fewer constrained degrees of freedom (dimensionality of the ground-state configuration manifold increases), the disordered direct-space configuration becomes less spatially correlated. For a fixed $N$ in the limit $\rho \rightarrow \infty$ (i.e., system volume $v_{F} \rightarrow 0$ ), every $\mathbf{k}$ point (except the origin) is expelled from the exclusion zone, and the system tends to an ideal-gas configuration [30], even if it is not an ideal gas thermodynamically, as shown in Sec. IV B.

states of matter. Hyperuniform systems are characterized by vanishing (normalized) density fluctuations at large length scales; i.e., the structure factor $S(\mathbf{k})$ tends to zero in the limit $|\mathbf{k}| \rightarrow 0$ [31] (see Sec. II A for details). The hyperuniformity concept provides a means of categorizing crystals, quasicrystals, and special disordered systems according to the degree to which large-scale density fluctuations are suppressed [31,32]. Disordered hyperuniform patterns, of which disordered stealthy systems are special cases, behave more like crystals in the manner they suppress large-scale density fluctuations, and yet they also resemble typical statistically isotropic liquids and glasses with no Bragg peaks. In this sense, they have a "hidden order" on large length scales that is not apparent at small length scales, even if short-range order is present; see Fig. 2 for a vivid illustration. During the last decade, a variety of disordered hyperuniform states have been identified that
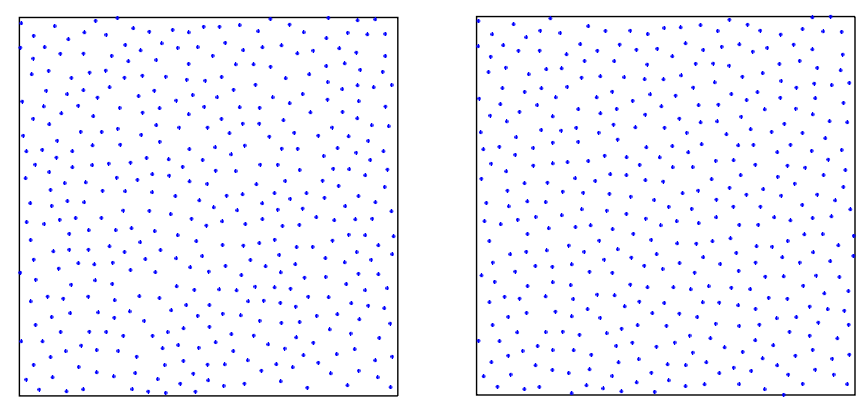

FIG. 2. A disordered nonhyperuniform configuration (left panel) and a disordered hyperuniform configuration (right panel). We arrive at the configuration on the right by very small collective displacements of the particles on the left via the methods described in Ref. [12]. (Each particle on average moves a root-mean-square distance that is about an order of magnitude smaller than the mean-nearest-neighbor distance as measured by the configuration proximity metric [53].) These two examples show that it can be very difficult to detect hyperuniformity by eye, and yet their large-scale density fluctuations are dramatically different ("hidden order").

exist as both equilibrium and nonequilibrium phases, including maximally random jammed particle packings [33-36], jammed athermal granular media [37], jammed thermal colloidal packings [38,39], cold atoms [40], transitions in nonequilibrium systems [41,42], surfaceenhanced Raman spectroscopy [43], terahertz quantum cascade laser [44], wave dynamics in disordered potentials based on supersymmetry [45], avian photoreceptor patterns [46], and certain Coulombic systems [47]. Moreover, disordered hyperuniform materials possess novel physical properties potentially important for applications in photonics [25-27,48,49] and electronics [50-52].

The well-known compressibility relation from statistical mechanics [54] provides some insights about the relationship between temperature $T$ and hyperuniformity for equilibrium systems at number density $\rho$ :

$$
S(k=0)=\rho k_{B} T \kappa_{T} .
$$

We see that any ground state $(T=0)$ in which the isothermal compressibility $\kappa_{T}$ is bounded and positive must be hyperuniform because the structure factor $S(k=$ $0)$ must be zero. This includes crystals as well as exotic disordered ground states such as stealthy ones. However, in order to have a hyperuniform system at positive $T$, the isothermal compressibility must be zero; i.e., the system must be incompressible [17] (see Refs. [31] and [47] for some examples). Subsequently, we will use relation (3) to draw some conclusions about the excited states associated with stealthy ground states.

Our general objective is the formulation of a predictive ensemble theory for the thermodynamic and structural properties of stealthy degenerate disordered ground states in arbitrary space dimension $d$ that complements previous 
numerical work on this topic [11-16]. After providing basic definitions and describing a family of isotropic stealthy potentials (Secs. II and III), we derive general exact relations for thermodynamic properties (energy, pressure, and isothermal compressibility) that apply to any welldefined ground-state ensemble as a function of the number density or, equivalently, $\chi$ in any space dimension $d$ (Sec. IV). We subsequently derive some exact integral conditions that both the pair correlation function $g_{2}(r)$ and structure factor $S(k)$ must obey (Sec. V). The existence of periodic stealthy ground states enables us to show how disordered degenerate ground states arise as part of the ground-state manifold for sufficiently small $\chi$ (Sec. VI). Subsequently, we derive analytical formulas for the pair statistics $\left[g_{2}(r)\right.$ and $\left.S(k)\right]$ for sufficiently small $\chi$ in the canonical ensemble in the limit that the temperature $T$ tends to zero (Sec. VII) by exploiting an ansatz that stealthy states behave like "pseudo"-equilibrium hard-sphere systems in Fourier space. Our theoretical predictions for $g_{2}(r)$ and $S(k)$ are in excellent agreement with computer simulations across the first three space dimensions. These results are then used to predict, with high accuracy, other structural characteristics of stealthy ground states across dimensions, such as order metrics, local number variance, and nearestneighbor functions (Secs. VIII and IX). Subsequently, we derive analytical formulas for the structure factor and thermal expansion coefficient for the associated excited states for sufficiently small temperatures (Sec. X). Finally, we provide concluding remarks in Sec. XI.

\section{DEFINITIONS AND PRELIMINARIES}

Roughly speaking, a point process in $d$-dimensional Euclidean space $\mathbb{R}^{d}$ is a distribution of an infinite number of points in $\mathbb{R}^{d}$ with the configuration $\mathbf{r}_{1}, \mathbf{r}_{2}, \ldots$ at a well-defined number density (number of points per unit volume). For a statistically homogeneous point process in $\mathbb{R}^{d}$ at number density $\rho$ [55], the quantity $\rho^{n} g_{n}\left(\mathbf{r}^{n}\right)$ is the probability density associated with simultaneously finding $n$ points at locations $\mathbf{r}^{n} \equiv \mathbf{r}_{1}, \mathbf{r}_{2}, \ldots, \mathbf{r}_{n}$ in $\mathbb{R}^{d}$ [54]. With this convention, each $n$-particle correlation function $g_{n}$ approaches unity when all of the points become widely separated from one another. Statistical homogeneity implies that $g_{n}$ is translationally invariant and hence only depends on the relative displacements of the positions with respect to any chosen system origin, e.g., $g_{n}=g_{n}\left(\mathbf{r}_{12}, \mathbf{r}_{13}, \ldots, \mathbf{r}_{1 n}\right)$, where $\mathbf{r}_{i j}=\mathbf{r}_{j}-\mathbf{r}_{i}$.

The pair correlation function $g_{2}(\mathbf{r})$ is a particularly important quantity. If the point process is also rotationally invariant (statistically isotropic), then $g_{2}$ depends on the radial distance $r \equiv|\mathbf{r}|$ only, i.e., $g_{2}(\mathbf{r})=g_{2}(r)$. Thus, it follows that the expected number of points $Z(R)$ found in a sphere of radius $R$ around a randomly chosen point of the point process, called the cumulative coordination function, is given by

$$
Z(R)=\rho s_{1}(1) \int_{0}^{R} x^{d-1} g_{2}(x) d x,
$$

where $s_{1}(r)=2 \pi^{d / 2} r^{d-1} / \Gamma(d / 2)$ is the surface area of a $d$-dimensional sphere of radius $r$. The total correlation function $h(\mathbf{r})$ is trivially related to $g_{2}(\mathbf{r})$ as follows:

$$
h(\mathbf{r}) \equiv g_{2}(\mathbf{r})-1 .
$$

When there are no long-range correlations in the system, $h(\mathbf{r}) \rightarrow 0$ or, equivalently, $g_{2}(\mathbf{r}) \rightarrow 1$ as $|\mathbf{r}| \rightarrow \infty$. The structure factor $S(\mathbf{k})$, which plays a prominent role in this paper, is related to the Fourier transform of $h(\mathbf{r})$, denoted by $\tilde{h}(\mathbf{k})$, via the expression

$$
S(\mathbf{k}) \equiv 1+\rho \tilde{h}(\mathbf{k})
$$

A lattice $\Lambda$ in $\mathbb{R}^{d}$ is a subgroup consisting of integer linear combinations of vectors that constitute a basis for $\mathbb{R}^{d}$, and thus, it represents a special subset of point processes. Here, the space can be geometrically divided into identical regions $F$ called fundamental cells, each of which contains just one point specified by the lattice vector

$$
\mathbf{p}=n_{1} \mathbf{a}_{1}+n_{2} \mathbf{a}_{2}+\cdots+n_{d-1} \mathbf{a}_{d-1}+n_{d} \mathbf{a}_{d},
$$

where $\mathbf{a}_{i}$ are the basis vectors for a fundamental cell and $n_{i}$ spans all the integers for $i=1,2, \ldots, d$. We denote by $v_{F}$ the volume of $F$. A lattice is called a Bravais lattice in the physical sciences. Unless otherwise stated, we will use the term lattice. Every lattice has a dual (or reciprocal) lattice $\Lambda^{*}$ in which the lattice sites are specified by the dual (reciprocal) lattice vector $\mathbf{q} \cdot \mathbf{p}=2 \pi m$ for all $\mathbf{p}$, where $m=0, \pm 1, \pm 2, \pm 3 \ldots$. The dual fundamental cell $F^{*}$ has volume $v_{F^{*}}=(2 \pi)^{d} / v_{F}$. This implies that the number density $\rho_{\Lambda}$ of $\Lambda$ is related to the number density $\rho_{\Lambda^{*}}$ of the dual lattice $\Lambda^{*}$ via the expression

$$
\rho_{\Lambda} \rho_{\Lambda^{*}}=1 /(2 \pi)^{d} .
$$

Some common $d$-dimensional lattices are mathematically defined in Appendix A.

A periodic point process (crystal) is a more general notion than a lattice because it is obtained by placing a fixed configuration of $N$ points (where $N \geq 1$ ) within a fundamental cell $F$ of a lattice $\Lambda$, which is then periodically replicated. Thus, the point process is still periodic under translations by $\Lambda$, but the $N$ points can occur anywhere in $F$; see Fig. 3.

\section{A. Hyperuniform point processes}

Consider uniformly sampling the number of points that are contained within a spherical window of radius $R$ of a point process in $\mathbb{R}^{d}$. A hyperuniform point process has the property that the local number variance $\sigma^{2}(R)$ grows more 


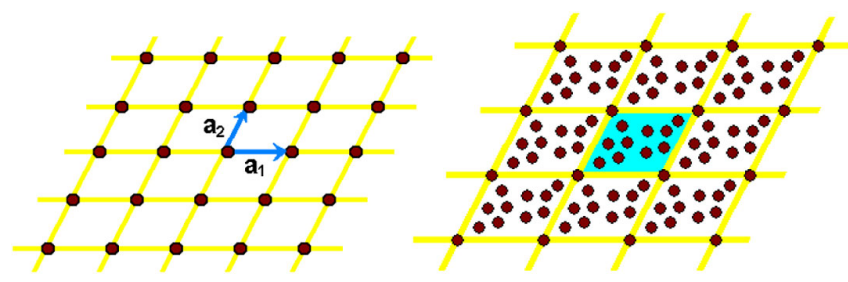

FIG. 3. (Bravais) lattice with one particle per fundamental cell (left panel) and a periodic crystal with multiple particles per fundamental cell (right panel).

slowly than $R^{d}$ [31]. Because $\sigma^{2}(R)$ is exactly related to a $d$-dimensional volume integral of the structure factor $S(\mathbf{k})$ (see Sec. VIII), this implies that hyperuniform states of matter possess infinite-wavelength density fluctuations (appropriately normalized) that vanish; i.e., $S(\mathbf{k})$ obeys the condition

$$
\lim _{|\mathbf{k}| \rightarrow 0} S(\mathbf{k}) \rightarrow 0,
$$

which means they are poised at an "inverted" critical point with associated scaling exponents [31]. For a Poisson (spatially uncorrelated) point process and many disordered point patterns, including typical liquids and structural glasses, the number variance grows like the volume of the window, i.e., $\sigma^{2}(R) \sim R^{d}$, implying that $S(\mathbf{k})$ is positive at $\mathbf{k}=\mathbf{0}$. All perfect crystals and quasicrystals are hyperuniform such that $\sigma^{2}(R) \sim R^{d-1}$; in other words, the variance grows like window surface area. By contrast, it is much more unusual to find disordered systems that are also hyperuniform. In recent years, evidence has been emerging that disordered hyperuniform many-particle systems can be regarded as new distinguishable states of disordered matter (see examples given in the Introduction). Whenever the structure factor goes to zero with the powerlaw form $S(\mathbf{k}) \sim|\mathbf{k}|^{\alpha}$, the number variance has the following large- $R$ asymptotic scaling that depends on the value of the exponent $\alpha[17,32]$ :

$$
\sigma^{2}(R) \sim\left\{\begin{array}{ll}
R^{d-1} \ln R & \alpha=1 \\
R^{d-\alpha} & \alpha<1 \\
R^{d-1} & \alpha>1
\end{array} \quad(R \rightarrow+\infty) .\right.
$$

Since disordered as well as ordered stealthy states can be viewed as systems in which $\alpha$ tends to infinity, we see from Eq. (10) that they have the asymptotic scaling $\sigma^{2}(R) \sim R^{d-1}$. We give theoretical predictions for the variance of disordered stealthy ground states in Sec. VIII.

\section{FAMILIES OF STEALTHY PAIR POTENTIALS}

As we see in the next section, the specific form of a stealthy potential does not affect the ground-state energy manifold, but it can affect other thermodynamic properties, such as the pressure. This has consequences in simulations of such properties, especially with respect to convergence issues. Hence, it is instructive to remark on some mathematical aspects of the long-range nature of the direct-space stealthy potentials, which are very similar to the weakly decaying Friedel oscillations of the electron density in a variety of systems, including molten metals as well as graphene [56,57]. As we will see, in some cases, stealthy potentials may mimic effective interactions that arise in certain polymer systems [10].

Here, we will limit ourselves to pair potentials $v(r)$ that are radial functions in $\mathbb{R}^{d}$, where $r=|\mathbf{r}|$ (i.e., isotropic pair interactions), and therefore, their Fourier transforms $\tilde{v}(k)$ are also radial functions in $\mathbb{R}^{d}$, where $k \equiv|\mathbf{k}|$ is a wave number. The $d$-dimensional Fourier transform of any integrable radial function $f(r)$ in $\mathbb{R}^{d}$ is given by [31]

$$
\tilde{f}(k)=(2 \pi)^{d / 2} \int_{0}^{\infty} r^{d-1} f(r) \frac{J_{(d / 2)-1}(k r)}{(k r)^{(d / 2)-1}} d r,
$$

and the inverse transform of $\tilde{f}(k)$ is given by

$$
f(r)=\frac{1}{(2 \pi)^{d / 2}} \int_{0}^{\infty} k^{d-1} \tilde{f}(k) \frac{J_{(d / 2)-1}(k r)}{(k r)^{(d / 2)-1}} d k,
$$

where $J_{\nu}(x)$ is the Bessel function of order $\nu$.

Consider the class of stealthy radial potential functions $\tilde{v}(k)$ in $\mathbb{R}^{d}$ that are bounded and positive with compact support in the radial interval $0 \leq k \leq K$, i.e.,

$$
\tilde{v}(k)=V(k) \Theta(K-k),
$$

where, for simplicity, $V(k)$ is infinitely differentiable in the open interval $[0, K)$ and

$$
\Theta(x)= \begin{cases}0 & x<0 \\ 1 & x \geq 0\end{cases}
$$

is the Heaviside step function. The corresponding directspace radial pair potential $v(r)$ is necessarily a delocalized, long-ranged function that is integrable in $\mathbb{R}^{d}$. Moreover, without any loss of generality, it will be assumed that $V(k) \leq v_{0}$.

For concreteness and purposes of illustration, we will examine properties of two specific families of potentials that fall within the aforementioned wide class of stealthy interactions: "power-law" and "overlap" potentials.

\section{A. Power-law potentials}

The power-law potentials are defined in Fourier space as follows:

$$
\tilde{v}(k)=v_{0}(1-k / K)^{m} \Theta(K-k),
$$

where the exponent $m$ can be any whole number. The corresponding direct-space potential $v(r)$ will depend on $d$ for any given $m$ and is exactly given by 


$$
\frac{v(r)}{v_{0}}=\frac{K^{d} \Gamma(m+1) \Gamma[(d+1) / 2] \cdot{ }_{1} F_{2}\left(a_{1} ; b_{1}, b_{2} ; x\right)}{\Gamma(1+m+d) \pi^{(d+1) / 2}},
$$

where $\quad a_{1}=(d+1) / 2, \quad b_{1}=1+(m+d) / 2, \quad b_{2}=$ $(1+m+d) / 2, x=-(K r)^{2} / 4$ and ${ }_{1} F_{2}\left(a_{1} ; b_{1}, b_{2} ; x\right)$ is a special case of the generalized hypergeometric function ${ }_{p} F_{q}\left(a_{1}, \ldots, a_{p} ; b_{1}, \ldots, b_{q} ; x\right)$ [58]. Because the potential (16) is derived from the Fourier power-law potential (15), we will refer to Eq. (16) as the direct-space power-law potential. In the instance when $m=0$ in Eq. (15) (i.e., simple step function), this expression for $v(r)$ simplifies as follows:

$$
\frac{v(r)}{v_{0}}=\left(\frac{K}{2 \pi r}\right)^{d / 2} J_{d / 2}(K r)
$$

for which the large- $r$ asymptotic behavior is given by

$$
\frac{v(r)}{v_{0}} \sim\left(\frac{K}{2 \pi}\right)^{(d-1) / 2} \frac{\cos (K r-(d+1) \pi / 4)}{\pi r^{(d+1) / 2}} \quad(r \rightarrow \infty) .
$$

For any fixed $d$ and $m \geq d$, the direct-space power-law potential has the asymptotic form

$$
\frac{v(r)}{v_{0}} \sim \frac{s(r ; m, d)}{r^{d+1}} \quad(r \rightarrow \infty),
$$

where $s(r ; m, d)$ is a bounded function (a sinusoidal function or constant of order one). For any fixed $d$ and $1 \leq m<d$, the long-range oscillations of $v(r)$ are controlled by an envelope that decays like $1 / r^{\beta}$, where $(d+1) / 2<\beta \leq d+1$.

In Fig. 4, we plot the Fourier power-law potential for selected values of $m$ (which applies in any dimension) and the corresponding direct-space potentials for $d=3$. In all cases, we set $v_{0}=K=1$. It is to be noted that the amplitudes of the oscillations in $v(r)$ decrease as $m$ increases for a fixed dimension.

\section{B. Overlap potentials}

Let $\alpha(r ; R)$ represent the intersection volume of two identical $d$-dimensional spheres of radius $R$ (scaled by the volume of sphere) whose centers are separated by a distance $r$. This quantity is known analytically in any space dimension and has a variety of representations [59], including the following:

$$
\alpha(r ; R)=c(d) \int_{0}^{\cos ^{-1}(r /(2 R))} \sin ^{d}(\theta) d \theta,
$$

where $c(d)$ is the $d$-dimensional constant given by

$$
c(d)=\frac{2 \Gamma(1+d / 2)}{\pi^{1 / 2} \Gamma((d+1) / 2)} .
$$

For $d=1,2,3$, and 4, we respectively have

$$
\begin{aligned}
& \alpha(r ; R)=\Theta(2 R-r)\left[1-\frac{r}{2 R}\right], \\
& \alpha(r ; R)=\Theta(2 R-r)\left[\frac{2}{\pi}\left(\cos ^{-1}\left(\frac{r}{2 R}\right)-\frac{r}{2 R}\left(1-\frac{r^{2}}{4 R^{2}}\right)^{1 / 2}\right)\right], \\
& \alpha(r ; R)=\Theta(2 R-r)\left[1-\frac{3}{4} \frac{r}{R}+\frac{1}{16}\left(\frac{r}{R}\right)^{3}\right], \\
& \alpha(r ; R)=\Theta(2 R-r)\left[\frac{2}{\pi}\left(\cos ^{-1}\left(\frac{r}{2 R}\right)-\left\{\frac{5 r}{6 R}-\frac{1}{12}\left(\frac{r}{R}\right)^{3}\right\}\left(1-\frac{r^{2}}{4 R^{2}}\right)^{1 / 2}\right)\right] .
\end{aligned}
$$
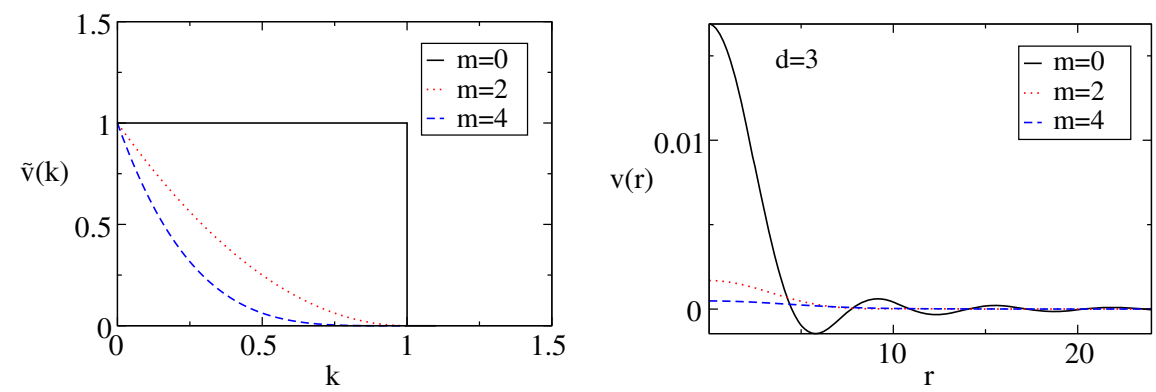

FIG. 4. Left panel: Fourier power-law potential $\tilde{v}(k)$ for the special cases $m=0,2$, and 4 that apply for any $d$. Right panel: Corresponding direct-space power-law potentials $v(r)$ in the instance $d=3$. Here, we set $v_{0}=K=1$. 

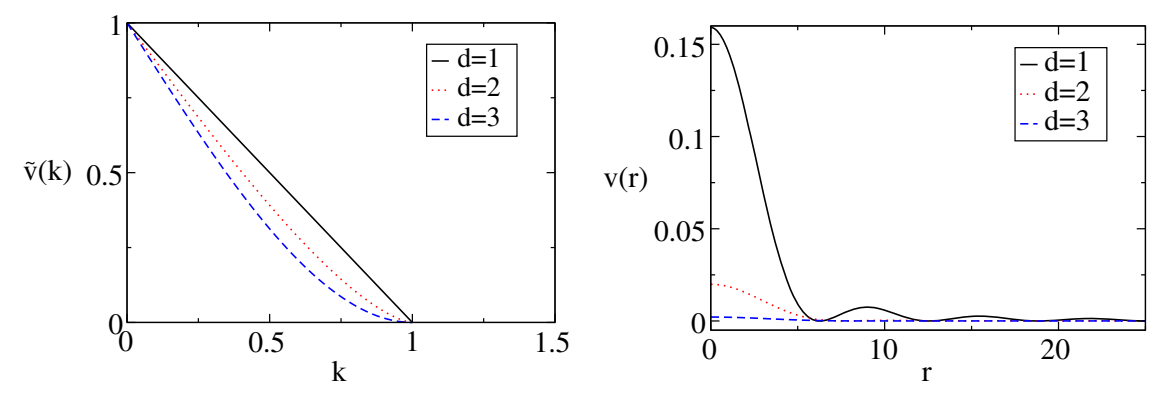

FIG. 5. Left panel: Fourier overlap potential $\tilde{v}(k)$ for the first three space dimensions. Right panel: Corresponding direct-space overlap potentials $v(r)$, which oscillate but are always non-negative. Here, we set $v_{0}=K=1$.

Consider the class of "overlap" potentials, which for any dimension is given by

$$
\tilde{v}(k)=v_{0} \alpha(r=k, R=K / 2) .
$$

Note that for $d=1$, the overlap potential is identical to the power-law potential when $d=1$ and $m=1$. The thermodynamics of the ground-state manifold of this potential in the special case $d=2$ was numerically investigated in Refs. [15] and [16]. It follows from Eq. (26) that the corresponding direct-space overlap potential is given by

$$
\frac{v(r)}{v_{0}}=\frac{\Gamma(1+d / 2)}{\pi^{d / 2}} \frac{J_{d / 2}^{2}(K r / 2)}{r^{d}},
$$

which is clearly non-negative for all $r$. Its large- $r$ asymptotic behavior is given by

$\frac{v(r)}{v_{0}} \sim \frac{4 \Gamma(1+d / 2)}{\pi^{d / 2+1}} \frac{\cos ^{2}(K r / 2-(d+1) \pi / 4)}{r^{d+1}} \quad(r \rightarrow \infty)$,

revealing that the long-ranged decay of the direct-space overlap potential has an envelope controlled by the inverse power law $1 / r^{d+1}$.

Figure 5 depicts the overlap potential $\tilde{v}(k)$ for the first three space dimensions and the corresponding direct-space overlap potentials $v(r)$, the latter of which vividly shows the increasing decay rate of $v(r)$ with increasing dimension. The direct-space overlap potential $v(r)$ is similar in functional form to effective positive pair interactions that arise in multilayered ionic microgels [10].

\section{ENSEMBLE THEORY FOR STEALTHY DISORDERED GROUND STATES: EXACT RESULTS FOR THERMODYNAMIC PROPERTIES}

Our general objective is the formulation of an ensemble theory for the thermodynamic and structural properties of stealthy degenerate disordered ground states that we previously investigated numerically [11-16]. In this section, we derive general exact relations for thermodynamic properties that apply to any well-defined ensemble as generated by a particular way to sample the stealthy disordered ground-state manifold as a function of number density $\rho$. In the subsequent section, we derive some exact results for the pair statistics for general ensembles.

\section{A. Preliminaries}

To begin, consider a configuration of $N$ identical particles with positions $\mathbf{r}^{N} \equiv \mathbf{r}_{1}, \ldots, \mathbf{r}_{N}$ in a large region of volume $V$ in $d$-dimensional Euclidean space $\mathbb{R}^{d}$. For particles interacting via a pair potential $v(\mathbf{r})$, the total potential energy $\Phi\left(\mathbf{r}^{N}\right)$ is given by

$$
\Phi\left(\mathbf{r}^{N}\right)=\sum_{i<j} v\left(\mathbf{r}_{i j}\right),
$$

where $\mathbf{r}_{i j}=\mathbf{r}_{j}-\mathbf{r}_{i}$. Of particular interest are classical ground states, i.e., those configurations that minimize the energy per particle $\Phi\left(\mathbf{r}^{N}\right) / N$.

The ensemble average of the energy (29) per particle $u$ in the thermodynamic limit can be written in terms of the pair correlation function $g_{2}(\mathbf{r})$ :

$$
\begin{aligned}
u \equiv\left\langle\frac{\Phi\left(\mathbf{r}^{N}\right)}{N}\right\rangle & =\frac{\rho}{2} \int_{\mathbb{R}^{d}} v(\mathbf{r}) g_{2}(\mathbf{r}) d \mathbf{r} \\
& =\frac{\rho}{2} \int_{\mathbb{R}^{d}} v(\mathbf{r}) d \mathbf{r}+\frac{\rho}{2} \int_{\mathbb{R}^{d}} v(\mathbf{r}) h(\mathbf{r}) d \mathbf{r},
\end{aligned}
$$

where angular brackets denote an ensemble average and $\rho$ is the number density in the thermodynamic limit.

Because the collective-coordinate approach relies on the Fourier representation of the energy, we recast Eq. (30) in terms of the structure factor $S(\mathbf{k})$ by applying Parseval's theorem to the second line of Eq. (30):

$$
\begin{aligned}
u & =\frac{\rho}{2} \tilde{v}(\mathbf{k}=\mathbf{0})+\frac{\rho}{2(2 \pi)^{d}} \int_{\mathbb{R}^{d}} \tilde{v}(\mathbf{k}) \tilde{h}(\mathbf{k}) d \mathbf{k} \\
& =\frac{\rho}{2} \tilde{v}(\mathbf{k}=\mathbf{0})-\frac{1}{2} v(\mathbf{r}=\mathbf{0})+\frac{1}{2(2 \pi)^{d}} \int_{\mathbb{R}^{d}} \tilde{v}(\mathbf{k}) S(\mathbf{k}) d \mathbf{k},
\end{aligned}
$$


where $\tilde{v}(\mathbf{k})$ and $\tilde{h}(\mathbf{k})$ are the Fourier transforms of $v(\mathbf{r})$ and $h(\mathbf{r})$, respectively, both of which are assumed to exist, $\mathbf{k}$ is a wave vector, and $S(\mathbf{k})$ is the structure factor defined in relation (6). Note that the structure factor is a non-negative, inversion-symmetric function, i.e.,

$$
S(\mathbf{k}) \geq 0 \quad \text { for all } \mathbf{k}, \quad S(\mathbf{k})=S(-\mathbf{k})
$$

\section{B. Ground-state energy and dimensionality of its configuration space}

Consider a radial (isotropic) stealthy potential function $\tilde{v}(k)$ with support in $0 \leq k \leq K$ of the class specified by Eq. (13). In light of Eq. (31), it is clear that whenever particle configurations in $\mathbb{R}^{d}$ exist such that $S(k)$ is constrained to achieve its minimum value of zero for $0 \leq k \leq K$, the system must be at its ground state or global energy minimum. This follows because the integrand $\tilde{v}(k) S(k)$ in the nontrivial term on the right side of Eq. (31) is identically zero because of the conflicting demands of the step functions. When such configurations exist, the average ground-state energy per particle in any well-defined ensemble is given exactly by

$$
u=\frac{\rho}{2} v_{0}-\frac{1}{2} v(r=0),
$$

which is a structure-independent constant, depending on the density $\rho, v_{0} \equiv \tilde{v}(k=0), v(r=0)$ and $d$, as explicitly shown below. Importantly, because $u$ is a constant independent of the structure, its value has no effect on the ground-state manifold, which is generally degenerate; hence, this manifold is invariant to the specific choice of the stealthy function $\tilde{v}(k)$ at fixed $\rho$ and $d$.

We would like to express the energy (33) in terms of the parameter $\chi$, defined by relation (2), which measures the relative number of independently constrained degrees of freedom for a finite system under periodic boundary conditions. Note that, in the thermodynamic limit, $M(K)$ in Eq. (2) is simply half of the volume of a sphere of radius $K$ [due to the inversion symmetry of $S(\mathbf{k})$ ] multiplied by the density $\rho_{\Lambda^{*}}$ of the dual lattice [cf. Eq. (8)], i.e.,

$$
M(K)=\rho_{\Lambda^{*}} \frac{v_{1}(K)}{2}=\frac{v_{1}(K)}{2(2 \pi)^{d} \rho},
$$

where we have used the fact that $\rho \rightarrow N \rho_{\Lambda}$ in this distinguished limit. Hence, from Eq. (2), we obtain the following expression for $\chi$ in the thermodynamic limit:

$$
\rho \chi=\frac{v_{1}(K)}{2 d(2 \pi)^{d}},
$$

where

$$
v_{1}(R)=\frac{\pi^{d / 2} R^{d}}{\Gamma(1+d / 2)}
$$

is the volume of a $d$-dimensional sphere (hypersphere) of radius $R$. We see that for fixed $K$ and $d$, which fixes the potential, $\chi$ is inversely proportional to $\rho$, which is the situation that we usually consider in this paper [60].

Hence, as $\chi$ tends to zero, $\rho$ tends to infinity, which configurationally corresponds counterintuitively to the uncorrelated ideal-gas limit (Poisson distribution), as discussed in the Introduction. As $\chi$ increases from zero, the density $\rho$ decreases and the dimensionality of the groundstate configuration manifold $D_{C}$ decreases. The configurational dimensionality per particle in the thermodynamic limit, $d_{C}$, can easily be obtained from the relation $D_{C}=$ $d N-2 M(K)$ for a finite system [61]; specifically,

$$
d_{C}=d(1-2 \chi)
$$

where $d_{C}=\lim _{D_{C} \rightarrow \infty, N \rightarrow \infty} D_{C} / N$.

Equations (33) and (35) yield the average ground-state energy per particle to be

$$
u=v_{0}\left[\frac{\rho}{2}-\gamma d \rho \chi\right], \quad\left(\rho_{\min }^{*} \leq \rho<\infty\right),
$$

where $\rho_{\min }^{*}$ is the minimal density associated with the dual of the densest Bravais lattice in direct space (as elaborated in Sec. VI), and

$$
\gamma=\frac{\int_{\mathbb{R}^{d}} \tilde{v}(k) d \mathbf{k}}{v_{0} v_{1}(K)}=\frac{(2 \pi)^{d} v(r=0)}{v_{0} v_{1}(K)}
$$

is a constant whose value depends on the specific form of the stealthy-potential class $\tilde{v}(k)$ defined by Eq. (13) and hence must lie in the interval $(0,1]$, where $\gamma=1$ corresponds to the step-function choice $\tilde{v}(k)=v_{0} \Theta(K-k)$. While the system in the limit $\chi \rightarrow 0(\rho \rightarrow \infty)$ corresponds configurationally to an ideal gas in so far as the pair correlation function is concerned, as we will explain in detail in Sec. VII A, thermodynamically, it is nonideal; see Eq. (38) for $u$ and Eq. (41) for the pressure.

\section{Energy route to pressure and isothermal compressibility}

The pressure in the thermodynamic limit at $T=0$ can be obtained from the energy per particle via the relation

$$
p=\rho^{2}\left(\frac{\partial u}{\partial \rho}\right)_{T} .
$$

Therefore, for stealthy potentials, we see from Eq. (33) that the ground-state pressure, for all possible values of $\rho$ or $\chi$, is given by the following simple expression: 


$$
p=\frac{\rho^{2}}{2} v_{0}, \quad\left(\rho_{\min }^{*} \leq \rho<\infty\right) .
$$

Hence, the isothermal compressibility $\kappa_{T} \equiv \rho^{-1}(\partial \rho / \partial p)_{T}$ of such a ground state is

$$
\kappa_{T}=\frac{v_{0}}{\rho^{2}}
$$

We see that as $\rho$ tends to infinity, the compressibility tends to zero.

Two important remarks are in order. First, estimates of the pressure obtained from simulations that we previously performed for $d=2[15,16]$, as well as those carried out in the present study across the first three space dimensions (Appendix B), are in very good agreement with the exact result (41) across a wide range of densities, thus validating the accuracy of the simulations. Second, the fact that the pressure (41) is a continuous function of density implies that any phase transition that may take place could be a continuous one, the implications of which are discussed in the Conclusions.

\section{Virial route to pressure and isothermal compressibility}

An alternative route to the pressure for a radial pair potential function $v(r)$ is through the "virial" equation, which at $T=0$ in the thermodynamic limit, is given by

$$
p=-\frac{\rho^{2}}{2 d} s_{1}(1) \int_{0}^{\infty} r^{d} \frac{d v}{d r} g_{2}(r) d r
$$

Although the pressure obtained via the virial route is generally expected to be equivalent to that obtained from the energy route (as described in the previous section), we will show that, for a certain class of stealthy potentials, the pressure obtained from Eq. (43) is either ill defined or divergent. This has practical implications for what types of stealthy potentials can be used in constant-pressure simulations.

It is convenient to rewrite the virial relation (43) in the following form:

$$
\begin{aligned}
p & =-\frac{\rho^{2}}{2 d}\left[\int_{\mathbb{R}^{d}} r \frac{d v}{d r} d \mathbf{r}+\int_{\mathbb{R}^{d}} r \frac{d v}{d r} h(r) d \mathbf{r}\right] \\
& =-\frac{\rho^{2}}{2 d}\left[\tilde{F}(k=0)+\frac{1}{(2 \pi)^{d}} \int_{\mathbb{R}^{d}} \tilde{F}(k) \tilde{h}(k) d \mathbf{k}\right],
\end{aligned}
$$

where $\tilde{F}(k)$ is the Fourier transform of $F(r) \equiv r d v / d r$, when it exists, and we have used Parseval's theorem and definition (5) for the total correlation function $h(r)$.

To continue with this analysis, we make use of the following lemma.
Consider a bounded radial function $\tilde{z}(k)$ with compact support on the radial interval $[0, K]$ in $\mathbb{R}^{d}$ that is infinitely differentiable in the open interval $[0, K)$. Therefore, its Fourier transform $z(r)$ exists.

Lemma 1.-The Fourier transform of the radial function $w(r)=r d z / d r$ in $\mathbb{R}^{d}$ is given by

$$
\tilde{w}(k)=-d \cdot \tilde{z}(k)-\frac{d \tilde{z}}{d k} .
$$

Proof.-Differentiation of $\tilde{z}(k)$ [defined via Eq. (11)] with respect to $k$ leads to the following identity:

$$
k \frac{d \tilde{z}}{d k}=-(2 \pi)^{d / 2} \int_{0}^{\infty} k r^{d} z(r) \frac{J_{(d / 2)}(k r)}{(k r)^{(d / 2)-1}} d r .
$$

The Fourier transform of $w(r)$ is given by

$$
\tilde{w}(k)=(2 \pi)^{d / 2} \int_{0}^{\infty} r^{d} \frac{d z}{d r} \frac{J_{(d / 2)-1}(k r)}{(k r)^{(d / 2)-1}} d r .
$$

Integrating relation (47) by parts and using Eq. (46) proves the lemma.

Corollary.-It immediately follows from Lemma 1 that $\tilde{w}(k)$ has the same support as $\tilde{z}(k)$ and

$$
\tilde{w}(k=0)=-d \cdot \tilde{z}(k=0),
$$

meaning that the volume integral of $r d z / d r$ over all space is proportional to the corresponding volume integral of $z(r)$.

Note that by the Corollary of Lemma 1, $\tilde{F}(0)=-d \cdot \tilde{v}(0)$, and hence we can rewrite the virial relation (44) as

$$
\begin{aligned}
p & =\frac{\rho^{2}}{2 d}\left[d \tilde{v}(k=0)+\frac{d}{(2 \pi)^{d}} \int_{\mathbb{R}^{d}} \tilde{F}(k) \tilde{h}(k) d \mathbf{k}\right] \\
& =\frac{\rho^{2}}{2 d}\left[d \tilde{v}(k=0)-\frac{d}{\rho(2 \pi)^{d}} \int_{\mathbb{R}^{d}} \tilde{F}(k) d \mathbf{k}\right] \\
& =\frac{\rho^{2}}{2} v_{0} .
\end{aligned}
$$

The second term in the second line of Eq. (49) follows because $\rho \tilde{h}(k)=-\Theta(K-k)$ inside the exclusion sphere of radius $K$ [see also Eq. (52) below] and has support in this exclusion zone by the Corollary of Lemma 1. But this second term must vanish in light of the trivial identity

$$
F(r=0)=\left(r \frac{d v}{d r}\right)_{r=0}=\frac{1}{(2 \pi)^{d}} \int_{\mathbb{R}^{d}} \tilde{F}(k) d \mathbf{k}=0 .
$$

We see that the virial ground-state pressure (49) agrees with that of the pressure obtained via the energy per particle [cf. Eq. (41)] provided that $\tilde{F}(k)$ exists. Since the latter is a 
stronger condition than the existence of $\tilde{v}(k)$, it is possible to devise a stealthy function $\tilde{v}(k)$ for which $\tilde{F}(k)$ does not exist and hence a virial pressure that either diverges or is nonconvergent. For example, this problem occurs for the power-law potential (15) with $m=0$ (step function) for any dimension $d$. By contrast, the virial pressure is always well defined for the overlap potential (26) in any dimension [62]. This example serves to illustrate the mathematical subtleties that can arise because of the long-ranged nature of stealthy potentials in direct space.

\section{ENSEMBLE THEORY FOR STEALTHY DISORDERED GROUND STATES: EXACT INTEGRAL CONDITIONS ON THE PAIR STATISTICS}

Here, we derive some exact integral conditions that must be obeyed by both the pair correlation function $g_{2}(r)$ and the structure factor $S(k)$ for stealthy ground states that apply to general ensembles. These analytical relations can be profitably employed to test corresponding computersimulation results.

\section{A. General properties}

In any stealthy ground state, the structure factor attains its minimum value $S(k)=0$ for $0<k \leq K$ and hence has the form

$$
S(k)=\Theta(k-K)[1+\tilde{Q}(k)],
$$

where $\Theta(x)$ is the Heaviside step function defined by Eq. (14) and $\tilde{Q}(k)=S(k)-1$ is a function that obeys the inequality $\tilde{Q}(k) \geq-1$. Therefore, from Eq. (6), we have that the Fourier transform of the total correlation function $h(r)$ has the form

$$
\rho \tilde{h}(k)=\tilde{f}(k)+\tilde{P}(k),
$$

where

$$
\tilde{f}(k)=-\Theta(K-k)
$$

and

$$
\tilde{P}(k)=\Theta(k-K) \tilde{Q}(k) .
$$

It is noteworthy that the function $\tilde{f}(k)$ is identical to the Mayer- $f$ function for an equilibrium hard-sphere system in direct space.

Taking the inverse Fourier transform of Eq. (52) yields the direct-space total correlation function, given by

$$
\rho h(r)=f(r)+P(r),
$$

where

$$
f(r)=-\left(\frac{K}{2 \pi r}\right)^{d / 2} J_{d / 2}(K r)
$$

and

$$
\begin{aligned}
P(r) & =\frac{1}{(2 \pi)^{(d / 2)}} \int_{K}^{\infty} k^{d-1} \tilde{Q}(k) \frac{J_{(d / 2)-1}(k r)}{(k r)^{(d / 2)-1}} d k \\
& \geq\left(\frac{K}{2 \pi r}\right)^{d / 2} J_{d / 2}(K r)-\rho,
\end{aligned}
$$

where the lower bound on $P(r)$ indicated in Eq. (57) follows from the fact that $h(r) \geq-1$ for all $r$ for any point pattern. It trivially follows that since $\rho \tilde{h}(k=0)=$ $\rho \int_{\mathbb{R}^{d}} h(r) d \mathbf{r}=-1$, the volume integral of $P(r)$ must be zero, i.e.,

$$
\int_{\mathbb{R}^{d}} P(r) d \mathbf{r}=0
$$

Less trivially, because the product $\tilde{v}(k) \tilde{P}(k)$ is zero for all $k$, by Parseval's theorem, we have the integral condition

$$
\int_{\mathbb{R}^{d}} v(r) P(r) d \mathbf{r}=0 .
$$

Thus, the functions $v(r)$ and $P(r)$ are orthogonal to one another. The exact integral conditions (58) and (59) can be used to test the accuracy of numerical methods that yield estimates of the pair correlation function.

\section{B. Behavior of the pair correlation function near the origin}

It is instructive to determine the behavior of the pair correlation function $g_{2}(r)$ for small $r$. Substitution of the general form (11) for $\tilde{h}(k)$ into the definition of the total correlation function $h(r)$ as obtained from Eq. (12), and expanding $h(r)$ in a Taylor series around $r=0$ through second order in the radial distance $r$, yields

$$
h(r)=h(r=0)+\frac{1}{2}\left(\frac{\partial^{2} h}{\partial r^{2}}\right)_{r=0} r^{2}+\mathcal{O}\left(r^{4}\right),
$$

where

$$
h(r=0)=-2 d \chi+2 d^{2} \chi \int_{K}^{\infty} k^{d-1} \tilde{Q}(k) d k,
$$

and the corresponding curvature is

$$
\left(\frac{\partial^{2} h}{\partial r^{2}}\right)_{r=0}=\frac{2 d}{d+2} \chi-2 d \chi \int_{K}^{\infty} k^{d+1} \tilde{Q}(k) d k .
$$

Therefore, from Eq. (61), we see that the pair correlation function at the origin is given by 


$$
g_{2}(r=0)=1-2 d \chi+2 d^{2} \chi \int_{K}^{\infty} k^{d-1} \tilde{Q}(k) d k .
$$

Since $g_{2}(r)$ must be non-negative for all $r$, we have the following integral condition on $\tilde{Q}(k)$ :

$$
2 d^{2} \chi \int_{K}^{\infty} k^{d-1} \tilde{Q}(k) d k \geq 2 d \chi-1 .
$$

Hence, this integral must be positive for

$$
\chi \geq \frac{1}{2 d}
$$

We also conclude from Eq. (62) that for $h(r)$ or $g_{2}(r)$ to have positive curvature at the origin, $\tilde{Q}(k)$ must obey the additional integral condition:

$$
(d+2) \int_{K}^{\infty} k^{d+1} \tilde{Q}(k) d k \leq 1 .
$$

Finally, we note that when $g_{2}(r=0)=0$, the results above yield the equality

$$
\int_{K}^{\infty} k^{d-1} \tilde{Q}(k) d k=\frac{2 d \chi-1}{2 d^{2} \chi},
$$

and, because the curvature must be positive in this instance, the inequality (66) must generally be obeyed.

The inequality (64), conditional inequality (66), and conditional equality (67) provide integral conditions to test the accuracy of numerical methods that yield estimates of the structure factor.

\section{EXISTENCE OF STEALTHY DISORDERED DEGENERATE GROUND STATES}

It is noteworthy that any periodic crystal with a finite basis is a stealthy ground state for all positive $\chi$ up to its corresponding maximum value $\chi_{\max }$ (or minimum value of the number density $\rho_{\min }$ ) determined by its first positive Bragg peak $\mathbf{k}_{\text {Bragg }}$ [minimal positive wave vector for which $S(\mathbf{k})$ is positive]. Tables I-IV list the pair $\chi_{\max }, \rho_{\min }$ for some common periodic patterns in one, two, three, and four dimensions, respectively, all of which are part of the ground-state manifold; see Appendix A for mathematical

TABLE I. Maximum values of $\chi$ and corresponding minimum values of $\rho$ for certain periodic stealthy ground states in $\mathbb{R}$ with $K=1$. The configuration with the largest possible value of $\chi_{\max }$ (smallest possible value of $\rho_{\min }$ ) corresponds to the integer lattice.

\begin{tabular}{lcc}
\hline \hline Structure & $\chi_{\max }$ & $\rho_{\min }$ \\
\hline Integer lattice $(\mathbb{Z})$ & 1 & $1 / 2 \pi=0.15915 \ldots$ \\
Periodic with $n$-particle basis & $1 / n$ & $n / 2 \pi=(0.15915 \ldots) n$ \\
\hline \hline
\end{tabular}

definitions. (The crystals denoted by $\mathrm{Dia}_{d}$ and $\mathrm{Kag}_{d}$ are $d$ dimensional generalizations of the diamond and kagomé crystals, respectively, for $d \geq 2$ [63].) While the mere existence of such periodic ground states does not provide any clues about their occurrence probability in some ensemble, we will use these results here to show how disordered degenerate ground states arise as part of the ground-state manifold for sufficiently small $\chi$.

At fixed $d$, the smallest value of $\rho_{\text {min }}$ listed in Tables I-IV, which we call $\rho_{\mathrm{min}}^{*}$, corresponds to the dual of the densest Bravais lattice in direct space and represents the critical density value below which a stealthy ground state does not exist for all $k \leq\left|\mathbf{k}_{\text {Bragg }}^{*}\right|$. The fact that $\rho_{\text {min }}^{*}$ corresponds to the body-centered-cubic (BCC) lattice for $d=3$ was initially shown analytically in Ref. [19] and subsequently numerically in Ref. [14]. We note that the values of $\rho_{\min }$ for the simple hexagonal lattice and hexagonal close-packed crystal for $d=3$ reported in Ref. [19] are incorrect because those calculations were based on the erroneous assumption that the structure factors at the corresponding shortest reciprocal lattice vectors have nonvanishing values.

Observe that in the case $d=1$, there is no non-Bravais lattice (periodic structure with a basis $n \geq 2$ ) for which $\chi_{\max }$ is greater than $1 / 2$, implying that the ground-state manifold is nondegenerate (uniquely the integer lattice) for $1 / 2<\chi \leq 1$. This case is to be contrasted with the cases $d \geq 2$ where the ground-state manifold must be degenerate [65] for $1 / 2<\chi<\chi_{\max }^{*}$ and nondegenerate only at the point $\chi=\chi_{\max }^{*}$, as implied by Tables II-IV. Here, $\chi_{\max }^{*}$ is the largest possible value of $\chi_{\max }$ in some fixed dimension.

Lemma.-At fixed $K$, a configuration comprised of the union (superposition) of $m$ different stealthy ground-state configurations in $\mathbb{R}^{d}$ with $\chi_{1}, \chi_{2}, \ldots, \chi_{m}$, respectively, is itself stealthy with a $\chi$ value given by

$$
\chi=\left[\sum_{i=1}^{m} \chi_{i}^{-1}\right]^{-1},
$$

which is the harmonic mean of the $\chi_{i}$ divided by $m$.

Proof.-Formula (68) is a direct consequence of the fact that $\chi$ is inversely proportional to the number density $\rho=$ $\sum_{i=1}^{m} \rho_{i}$ of the union of the configurations in $\mathbb{R}^{d}$, where $\rho_{i}$ is the number density associated with the $i$ th configuration, which is inversely proportional to $\chi_{i}$.

This Lemma, together with the fact that any periodic crystal with a finite basis is a stealthy ground state, can be used to demonstrate rigorously how complex aperiodic patterns can be ground states, entropically favored or not. A sketch of such a proof would involve the consideration of the union of $m$ different periodic structures in $\mathbb{R}^{d}$ with densities $\rho_{1}, \rho_{2}, \ldots, \rho_{m}$, respectively, each of which are randomly translated and oriented with respect to some coordinate system such that $m$ is very large but bounded and $\rho_{i} \neq \rho_{j}$ for all $i$ and $j$. It is clear that the resulting configuration will be a highly complex aperiodic structure 
TABLE II. Maximum values of $\chi$ and corresponding minimum values of $\rho$ for certain periodic stealthy ground states in $\mathbb{R}^{2}$ with $K=1$. The configuration with the largest possible value of $\chi_{\max }$ (smallest possible value of $\rho_{\min }$ ) corresponds to the triangular lattice.

\begin{tabular}{lcc}
\hline \hline Structure & $\chi_{\max }$ & $\rho_{\min }$ \\
\hline Kagomé crystal $\left(\mathrm{Kag}_{2}\right)$ & $\pi / 3 \sqrt{12}=0.3022 \ldots$ & $3 \sqrt{3} / 8 \pi^{2}=0.06581 \ldots$ \\
Honeycomb crystal $\left(\mathrm{Dia}_{2}\right)$ & $\pi / 2 \sqrt{12}=0.4534 \ldots$ & $\sqrt{3} / 4 \pi^{2}=0.04387 \ldots$ \\
Square lattice $\left(\mathbb{Z}^{2}=\mathbb{Z}_{*}^{2}\right)$ & $\pi / 4=0.7853 \ldots$ & $1 / 4 \pi^{2}=0.02533 \ldots$ \\
Triangular lattice $\left(A_{2} \equiv A_{2}^{*}\right)$ & $\pi / \sqrt{12}=0.9068 \ldots$ & $\sqrt{3} / 8 \pi^{2}=0.02193 \ldots$ \\
\hline \hline
\end{tabular}

TABLE III. Maximum values of $\chi$ and corresponding minimum values of $\rho$ for certain periodic stealthy ground states in $\mathbb{R}^{3}$ with $K=1$. Here, MCC refers to the mean-centered cuboidal lattice, which is a Bravais lattice intermediate between the BCC and FCC lattices and has an equivalent dual lattice [64]. The configuration with the largest possible value of $\chi_{\max }$ (smallest possible value of $\rho_{\min }$ ) corresponds to the $\mathrm{BCC}$ lattice.

\begin{tabular}{lcc}
\hline \hline Structure & $\chi_{\max }$ & $\rho_{\min }$ \\
\hline Pyrochlore crystal $\left(\mathrm{Kag}_{3}\right)$ & $\pi / 4 \sqrt{12}=0.2267 \ldots$ & $2 / 3 \sqrt{3} \pi^{3}=0.01241 \ldots$ \\
Diamond crystal $\left(\mathrm{Dia}_{3}\right)$ & $\pi / 2 \sqrt{12}=0.4534 \ldots$ & $1 / 3 \sqrt{3} \pi^{3}=0.00620 \ldots$ \\
Simple hexagonal lattice & $\sqrt{3} \pi / 9=0.6045 \ldots$ & $1 / 4 \sqrt{3} \pi^{3}=0.00465 \ldots$ \\
SC lattice $\left(Z_{3} \equiv Z_{3}^{*}\right)$ & $2 \pi / 9=0.6981 \ldots$ & $1 / 8 \pi^{3}=0.00403 \ldots$ \\
HCP crystal & $8 \sqrt{6} \pi / 81=0.7600 \ldots$ & $3 \sqrt{3} / 32 \sqrt{2} \pi^{3}=0.00370 \ldots$ \\
FCC lattice $\left(D_{3} \equiv A_{3}\right)$ & $\pi / \sqrt{12}=0.9068 \ldots$ & $1 / 6 \sqrt{3} \pi^{3}=0.00310 \ldots$ \\
MCC lattice & $0.9258 \ldots$ & $0.00303 \ldots$ \\
BCC lattice $\left(D_{3}^{*} \equiv D_{3}^{*}\right)$ & $2 \sqrt{2} \pi / 9=0.9873 \ldots$ & $1 / 8 \sqrt{2} \pi^{3}=0.00285 \ldots$ \\
\hline \hline
\end{tabular}

TABLE IV. Maximum values of $\chi$ and corresponding minimum values of $\rho$ for certain periodic stealthy ground states in $\mathbb{R}^{4}$ with $K=1$. The configuration with the largest possible value of $\chi_{\max }$ (smallest possible value of $\rho_{\min }$ ) corresponds to the fourdimensional checkerboard lattice $D_{4} \equiv D_{4}^{*}$.

\begin{tabular}{lcc}
\hline \hline Structure & \multicolumn{1}{c}{$\chi_{\max }$} & $\rho_{\min }$ \\
\hline $\mathrm{Kag}_{4}$ crystal & $\pi^{2} / 40=0.2467 \ldots$ & $5 / 32 \pi^{3}=0.001640 \ldots$ \\
Dia $_{4}$ crystal & $\pi^{2} / 16=0.6168 \ldots$ & $1 / 16 \pi^{3}=0.0006416 \ldots$ \\
$\mathbb{Z}^{4}$ lattice & $\pi^{2} / 16=0.6168 \ldots$ & $1 / 16 \pi^{3}=0.0006416 \ldots$ \\
$D_{4}$ lattice & $\pi^{2} / 8=1.2337 \ldots$ & $1 / 32 \pi^{3}=0.0003208 \ldots$ \\
\hline \hline
\end{tabular}

in $\mathbb{R}^{d}$ that tends toward a disordered stealthy pattern with a value of $\chi$ that is very small but positive according to relation (68).

\section{PAIR STATISTICS IN THE CANONICAL ENSEMBLE: "PSEUDO” HARD SPHERES IN FOURIER SPACE}

The task of formulating an ensemble theory that yields analytical expressions for the pair statistics of stealthy degenerate ground states is highly nontrivial because the dimensionality of the configuration space depends on the density (or $\chi$ ) and there is a multitude of ways of sampling the ground-state manifold, each with its own probability measure for finding a particular ground-state configuration. Therefore, it is desirable to specialize to equilibrium ensembles with Gibbs measures because the characterization of the ground states (as well as the corresponding excited states) would be most tractable theoretically. In particular, our objective is to derive analytical formulas for the pair statistics of stealthy disordered ground states for sufficiently small $\chi$ in the canonical ensemble as temperature $T$ tends to zero; i.e., the probability of observing a configuration is proportional to $\exp \left[-\Phi\left(\mathbf{r}^{N}\right) /\left(k_{B} T\right)\right]$ in the limit $T \rightarrow 0$. We show here that under such circumstances, the pair statistics in the thermodynamic limit can be derived under the ansatz that stealthy ground states behave remarkably like pseudoequilibrium hard-sphere systems in Fourier space. This ansatz enables us to exploit well-known accurate expressions for the pair statistics in direct space. As will be shown, agreement with computer simulations is excellent for sufficiently small $\chi$.

\section{A. Pseudo-hard-sphere ansatz}

We have already noted that the step-function contribution to $\rho \tilde{h}(k)$ for stealthy ground states, denoted by $\tilde{f}(k)$ in relation (52), is identical to the Mayer- $f$ function for an equilibrium hard-sphere system in direct space. This implies that the corresponding contribution to $S(k)$ is a simple hard-core step function $\Theta(k-K)$, which can be viewed as an equilibrium hard-sphere system in Fourier space with "spheres" of diameter $K$ in the limit that $\chi$ tends to zero. Why is this the case? Because such a step function is exactly the same as the pair correlation $g_{2}(r=k)$ of an equilibrium hard-sphere system in direct space in the limit 
that $\rho$ tends to zero. That the structure factor must have the behavior $S(k) \rightarrow \Theta(k-K)$ in the limit $\chi \rightarrow 0$ is perfectly reasonable since a perturbation about the ideal-gas limit [where $S(k)=1$ for all $k$ ] in which an infinitesimal fraction of the degrees of freedom are constrained should only introduce an infinitesimal change in $S(k)$ of zero inside the exclusion zone (constrained region). We call this the weakly constrained limit, where a step function $S(k)$ is expected on maximum entropy grounds; it corresponds to the most disordered (decorrelated) form of $S(k)$ subject to the impenetrability condition in Fourier space. We refer to this phenomenon as equilibrated pseudo hard spheres in Fourier space because there are actually no points in that space that have a hard-core repulsion like true hard spheres do in direct space.

On the same maximum entropy grounds, we expect that a perturbation expansion about the weakly constrained limit $\chi=0$ will lead to a perturbation expansion in $\chi$ for $S(k)$ that can be mapped to the low-density expansion of $g_{2}(r)$ for equilibrium hard spheres. More generally, we make the ansatz that, in the canonical ensemble as $T \rightarrow 0$, this hardsphere analogy continues to hold as $\chi$ is increased from zero to positive values, provided that $\chi$ is small enough, implying that the collective coordinate variables $\tilde{n}(k)$ (defined in the Introduction) are weakly correlated. Though the pseudo-hard-sphere picture must break down in some intermediate range of $\chi$, for $d=1$ and $d=2$, this hard-sphere mapping is again exact when $\chi=\chi_{\max }$, which corresponds to the maximal value of the packing fraction $\eta$ in these dimensions (see Tables I and II). This exact correspondence with the maximal value of $\eta$ when $\chi=$ $\chi_{\max }$ does not hold for $d=3$ or $d=4$, however. Thus, one should only expect that $\chi$ and $\eta$ are proportional to one another, even at small $\chi$ values.

Under the pseudo-hard-sphere ansatz, the direct-space pair correlation function $g_{2}^{H S}(r ; \eta)$ of a disordered hardsphere system at a packing fraction $\eta$ for sufficiently small $\eta$ can be mapped into the structure factor $S(k ; \chi)$ for a disordered stealthy ground state derived from the canonical ensemble at fixed $\chi$ for sufficiently small $\chi$ as follows:

$$
S(k ; \chi)=g_{2}^{H S}(r=k ; \eta) .
$$

As alluded to above, the parameter $\chi$ can be viewed as an effective packing fraction for pseudo hard spheres of diameter $K$ in reciprocal space that is proportional to $\eta$, i.e.,

$$
\eta=b(d) \chi,
$$

where $b(d)$ is a $d$-dependent parameter that is to be determined. Let $h_{H S}(r)$ be the total correlation function of a disordered equilibrium hard-sphere system in direct space and let us define, for stealthy ground states,

$$
\tilde{H}(k) \equiv S(k)-1=\rho \tilde{h}(k) .
$$

The ansatz is also defined by the alternative mapping

$$
\tilde{H}(k)=h_{H S}(r=k) .
$$

This mapping then enables us to exploit the well-known statistical-mechanical theory of equilibrium hard-sphere systems. In particular, we can employ a generalized Ornstein-Zernike convolution relation that defines the appropriate direct correlation function $\tilde{C}(k)$, namely,

$$
\tilde{H}(k)=\tilde{C}(k)+\eta \tilde{H}(k) \otimes \tilde{C}(k),
$$

where the symbol $\otimes$ denotes the convolution operation in $\mathbb{R}^{d}$. Therefore, in direct space, $H(r)$ is given by the relation of the following form:

$$
H(r)=\frac{C(r)}{1-(2 \pi)^{d} \eta C(r)} .
$$

For example, for $d=1$,

$$
\tilde{C}(k)=-\Theta(1-k) \frac{(1-\eta k)}{(1-\eta)^{2}} .
$$

Inverting this function yields

$$
C(r)=\frac{-r \sin (r)+(r[\sin (r)+\cos (r)]-1) \eta}{\pi r^{2}(1-\eta)^{2}} .
$$

For $d=2$ and $d=3$, one can use the Percus-Yevick closure of the Ornstein-Zernike integral equation [54], which is highly accurate for low to intermediate densities along the liquid branch, or when mapped to the stealthy problem, for low to intermediate values of $\chi$.

It is noteworthy that the exact low-density expansion of $h_{H S}(r)$, for practical purposes, is sufficient to produce accurate estimates of $\tilde{H}(k)=\rho \tilde{h}(k)$ and its counterpart $\rho h(r)$ for low to intermediate values of $\chi$ or $\eta$. In particular, using the mapping (72), we obtain, for any dimension $d$, the following low- $\chi$ expansion of $\rho \tilde{h}(k)$ :

$$
\rho \tilde{h}(k)=-\Theta(K-k)\left[1+2^{d} b(d) \alpha(k ; K) \chi+\mathcal{O}\left(\chi^{2}\right)\right],
$$

where $b(d)$ is the proportionality constant in Eq. (70) and $\alpha(k ; K)$ is the scaled intersection volume of two identical $d$ dimensional spheres of diameter $K$ whose centers are separated by a distance $k$ [cf. Eq. (20)] [66]. This formula indicates that $S(k)$ develops a peak value at $k=K$ (over and above the value of unity due to the step function in the limit $\chi \rightarrow 0$ ) and then monotonically decreases until $k=2 K$, where it achieves its long-range value of unity for all $k>2 K$, which we will see is verified by computer simulations. Fourier inversion of Eq. (77), division by $\rho$, and use of (35) yields a corresponding low- $\chi$ expansion of 
the total correlation function $h(r)$ through second order in $\chi$ and hence has an error term of order $\chi^{3}$.

To get an idea of the large- $r$ asymptotic behavior of the pair correlations, consider the limit $\chi \rightarrow 0$ for any $d$. In this limit, the total correlation function $h(r)$ for any $r$ obtained from Eq. (77) is given by

$$
\rho h(r)=-\left(\frac{K}{2 \pi r}\right)^{d / 2} J_{d / 2}(K r) \quad(\chi \rightarrow 0),
$$

which for large $r$ is given asymptotically by

$$
\rho h(r) \sim-\frac{1}{r^{(d+1) / 2}} \cos [r-(d+1) \pi / 4] \quad(r \rightarrow+\infty) .
$$

Thus, the longed-ranged oscillations of $h(r)$ are controlled by the power law $-1 / r^{(d+1) / 2}$. Equation (78) indicates that in the limits $\chi \rightarrow 0$ and $\rho \rightarrow \infty, h(r) \rightarrow 0$, and therefore, the pair correlation function tends to the ideal gas even though the structure factor [Eq. (69)] cannot tend to the ideal-gas form because of its stealthy property. This result is in contrast to the situation considered in Fig. 1, where we take the $\rho \rightarrow \infty$ limit by fixing $N$ and letting $v_{F} \rightarrow 0$. In that case, both $g_{2}(r)$ and $S(k)$ tend to the associated idealgas forms, i.e., $g_{2}(r)=1$ for all $r$ and $S(k)=1$ for all $k$.

\section{B. Comparison of theoretical predictions to simulations}

In order to test our theoretical results for the pair statistics of stealthy ground states in the canonical ensemble, we have carried out computer simulations to generate and sample such configurations, the details of which are described in Appendix B. In all cases, we take $K=1$, which sets the length scale. Our simulation results reveal that the functional trends for $S(k)$ and $g_{2}(r)$ predicted by the ansatz of pseudo hard spheres in Fourier space with an effective packing fraction $\chi$ are remarkably accurate for a moderate range of $\chi$ about $\chi=0$. Because it is theoretically highly challenging to ascertain the proportionality constant $b(d)$ in Eq. (70) that arises in Eq. (77), we must rely on the simulations to guide us in its determination. First, we observe that for $d=1$, the mapping between $\chi$ and $\eta$ is one to one, i.e., $b(1)=1$. Second, the simulation data suggest that, to an excellent approximation, $b(d)$ for $d \geq 2$ is given by assuming that the peak value of $S(k)$ or $\rho \tilde{h}(k)$, achieved at $k=K$ for sufficiently small $\chi$, is invariant with respect to this peak value as in the one-dimensional case, and consequently $b(d)=\left[\alpha(K ; K) 2^{d}\right]^{-1}$.

Figure 6 shows that the structure factor $S(k)$, as obtained from Eqs. (71) and (77), is in excellent agreement with the corresponding simulated quantities for $\chi=0.05,0.1$, and 0.143 for $d=3$. In Fig. 7, we compare our theoretical results for the pair correlation function $g_{2}(r)$, as obtained by Fourier inversion of Eq. (77), to corresponding simulation results across the first three space dimensions. Again, we see excellent agreement between theory and
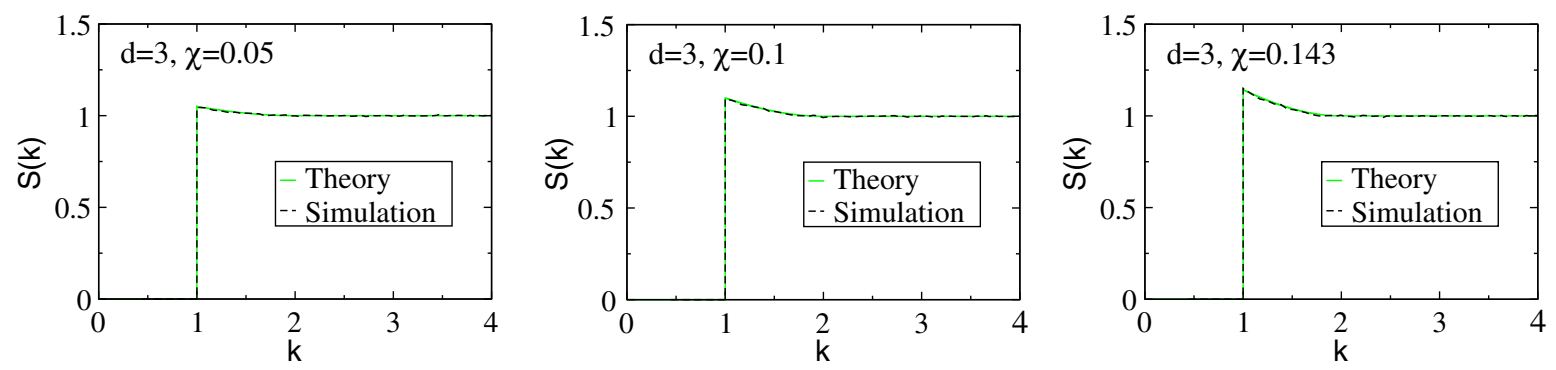

FIG. 6. Comparison of theoretical and simulation results for the structure factor $S(k)$ for $\chi=0.05,0.1$, and 0.143 for $d=3$. Here, $K=1$.
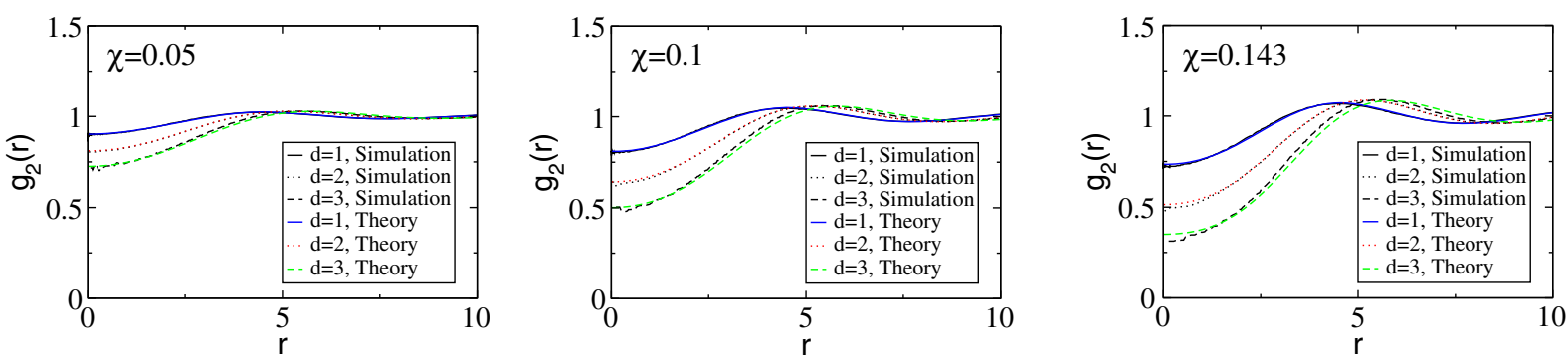

FIG. 7. Comparison of theoretical and simulation results for the pair correlation function $g_{2}(r)$ for $\chi=0.05,0.1$, and 0.143 across the first three space dimensions. Here, $K=1$. 


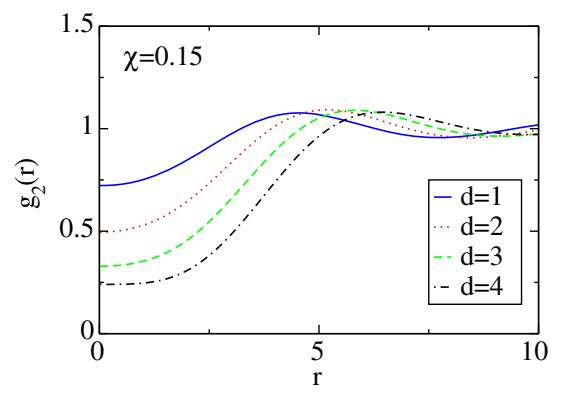

FIG. 8. Theoretical predictions for the pair correlation function $g_{2}(r)$ for $\chi=0.15$ across the first four space dimensions. Here, $K=1$.

simulations, which validates the pseudo-hard-sphere Fourier-space ansatz. Figure 8 depicts our theoretical predictions for $g_{2}(r)$ for $\chi=0.15$ across the first four space dimensions. It is seen that increasing dimensionality increases short-range correlations.

\section{Translational order or disorder metric}

We have seen that both short- and long-scale correlations increase as $\chi$ increases. A useful scalar positive order metric that captures the degree to which translational order increases with $\chi$ is given by

$$
\begin{aligned}
\tau & \equiv \frac{1}{D^{d}} \int_{\mathbb{R}^{d}} h^{2}(r) d \mathbf{r} \\
& =\frac{1}{(2 \pi)^{d} D^{d}} \int_{\mathbb{R}^{d}} \tilde{h}^{2}(k) d \mathbf{k},
\end{aligned}
$$

where we have used Parseval's theorem and $D$ is some characteristic length scale [68]. Note that for an ideal gas (spatially uncorrelated Poisson point process), $\tau=0$ because $h(r)=0$ for all $r$. Thus, a deviation of $\tau$ from zero measures translational order with respect to the fully uncorrelated case. Because $\tau$ diverges for any perfect crystal, it is a quantity that is better suited to distinguish the degree of pair correlations in amorphous systems.

In the case of stealthy ground-state configurations, $\tau$ is given explicitly by the relation

$$
\tau=\frac{1}{(2 \pi)^{d} \rho^{2} D^{d}} \int_{\mathbb{R}^{d}} \tilde{H}^{2}(k) d \mathbf{k},
$$

where $\tilde{H}(k)$ is given by Eq. (71). Substitution of the leading-order term in the $\chi$ expansion (77) into Eq. (81) yields

$$
\tau=\frac{4 d^{2}(2 \pi)^{d}}{v_{1}(1)} \chi^{2}+\mathcal{O}\left(\chi^{3}\right)
$$

where we have taken $D=K^{-1}$. Thus, for stealthy ground states, the order metric $\tau$ grows quadratically with $\chi$ for small $\chi$. Since the error is of order $\chi^{3}$, we expect that this quadratic form will be a very good approximation of $\tau$ up to moderately large values of $\chi$. Indeed, this is confirmed by our simulations up to $\chi=0.25$. Note that because stealthy disordered ground states (for sufficiently small $\chi$ ) are pseudo-equilibrium hard-sphere systems in Fourier space, the form of the order metric $\tau$ [Eq. (81)] ensures that it will behave similarly to $\tau$ for equilibrium hard spheres in direct space for low densities.

\section{LOCAL NUMBER VARIANCE FOR STEALTHY HYPERUNIFORM DISORDERED GROUND STATES}

Here, we investigate theoretically the local number variance for stealthy disordered ground states as a function of $\chi$ and then use these results to extract an order metric [31] that describes the extent to which large-scale density fluctuations are suppressed as $\chi$ increases in these hyperuniform systems (see Sec. II A). The local number variance $\sigma^{2}(R)$ associated with a general statistically homogeneous and isotropic point process in $\mathbb{R}^{d}$ at number density $\rho$ for a spherical window of radius $R$ is determined entirely by pair correlations [31]:

$$
\begin{aligned}
\sigma^{2}(R) & =\rho v_{1}(R)\left[1+\rho \int_{\mathbb{R}^{d}} h(r) \alpha(r ; R) d \mathbf{r}\right] \\
& =\rho v_{1}(R)\left[\frac{1}{(2 \pi)^{d}} \int_{\mathbb{R}^{d}} S(k) \tilde{\alpha}(k ; R) d \mathbf{k}\right],
\end{aligned}
$$

where $v_{1}(R)$ is the $d$-dimensional volume of a spherical window [cf. Eq. (36)], $h(r)$ is the total correlation function [cf. Eq. (5)], $\alpha(r ; R)$ is the scaled intersection volume of two spherical windows of radius $R$, as given by Eq. (20), and $\tilde{\alpha}(k ; R)$ is the Fourier transform of $\alpha(r ; R)$, which is explicitly given by [31]

$$
\tilde{\alpha}(k ; R)=2^{d} \pi^{d / 2} \Gamma(1+d / 2) \frac{\left[J_{d / 2}(k R)\right]^{2}}{k^{d}} .
$$

We have already noted that the stealthy ground states considered in the present paper are hyperuniform, i.e., $S(k) \rightarrow 0$ as $k \rightarrow 0$ (see Sec. II A). This means that such systems obey the sum rule $\rho \int_{\mathbb{R}^{d}} h(r) d \mathbf{r}=-1$ and, because of the rapid manner in which $S(k)$ vanishes in the limit $k \rightarrow 0$, the number variance has the following large- $R$ asymptotic behavior [31]:

$$
\sigma^{2}(R)=\Lambda(R) R^{d-1}+\mathcal{O}\left(R^{d-3}\right),
$$

where $\Lambda(R)$ is a bounded function that oscillates around an average value

$$
\bar{\Lambda}=\lim _{L \rightarrow \infty} \frac{1}{L} \int_{0}^{L} \Lambda(R) d R
$$



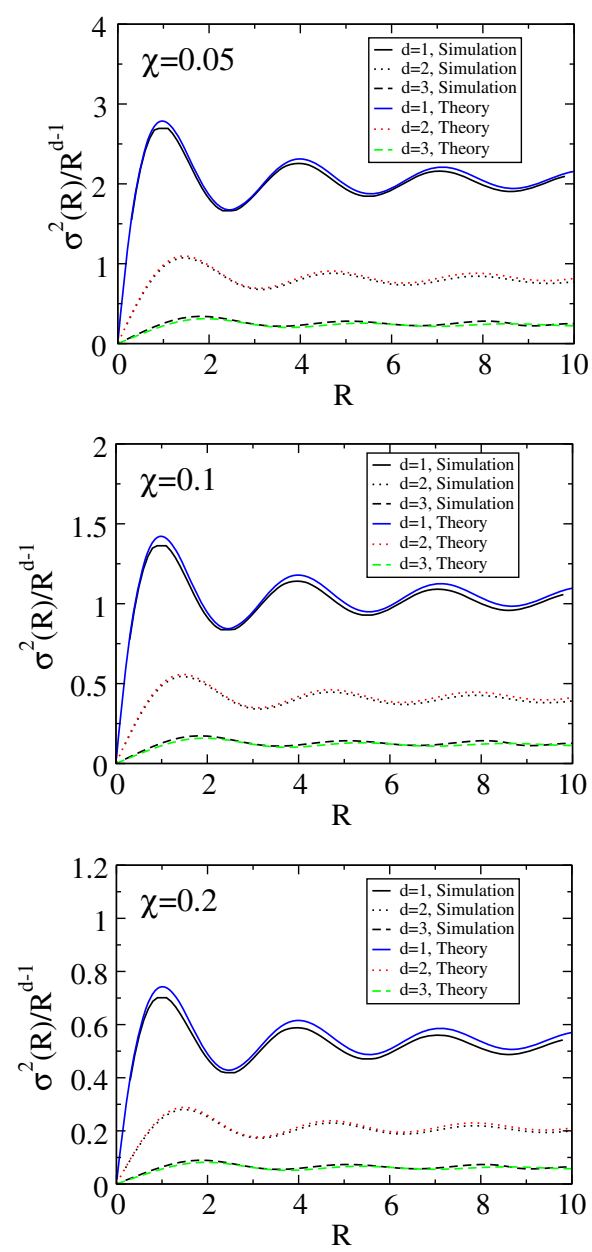

FIG. 9. Comparison of analytical and numerical results for the number variance for $\chi=0.05,0.1$, and 0.2 across the first three space dimensions. Here, $K=1$.

The scaling (85) occurs for a broader class of hyperuniform systems, as specified by relation (10). The parameter $\bar{\Lambda}$ is an order metric that quantifies the extent to which largescale density fluctuations are suppressed in such hyperuniform systems [31]. To compare different hyperuniform systems, Torquato and Stillinger used the following rescaled order metric:

$$
\bar{B}=\frac{\bar{\Lambda}}{\phi^{(d-1) / d}},
$$

which is independent of the density, where $\phi=\rho v_{1}(1 / 2)$. Among all hyperuniform point patterns having the scaling (85), $\bar{B}$ is minimized (greatest suppression of large-scale density fluctuations) for the integer, triangular, BCC, and $D_{4}$ lattices for $d=1,2,3$, and 4, respectively [31,32].

Using the analytical results for $g_{2}(r)$ or $S(k)$ described in the previous section, we have computed relation (83) for $\sigma^{2}(R)$ versus $R$ for selected values of $\chi$ across the first three dimensions and compared them to our corresponding simulation results; see Fig. 9. The analytical and numerical
TABLE V. The order metric $\bar{B}$ for various values of $\chi$ across the first four dimensions for disordered stealthy ground states for selected values of $\chi$ up to $\chi=0.25$, as obtained from the analytical estimates of $\sigma^{2}(R)$ and Eq. (87). Included for comparison are the structures in each dimension that have the minimal values of $\bar{B}$, all of which are Bravais lattices $[31,32]$.

\begin{tabular}{lllll}
\hline \hline$\chi$ & $d=1$ & $d=2$ & $d=3$ & $d=4$ \\
\hline 0.05 & 2.071 & 1.452 & 2.164 & 4.560 \\
0.1 & 1.051 & 1.040 & 1.738 & 3.875 \\
0.143 & 0.745 & 0.880 & 1.558 & 3.576 \\
0.2 & 0.54 & 0.755 & 1.411 & 3.327 \\
0.25 & 0.439 & 0.683 & 1.325 & 3.179 \\
Integer lattice & 0.167 & & & \\
Triangular lattice & & 0.508 & & \\
BCC lattice & & & 1.245 & \\
$D_{4}$ lattice & & & & 2.798 \\
\hline \hline
\end{tabular}

results are in excellent agreement with one another. Table $\mathrm{V}$ lists the order metric $\bar{B}$ for various values of $\chi$ across the first four dimensions for disordered stealthy ground states, as obtained from the analytical estimates of $\sigma^{2}(R)$ and Eq. (87). These results are also compared to the corresponding optimal values. As expected, $\bar{B}$ decreases as $\chi$ increases for fixed $d$.

\section{NEAREST-NEIGHBOR FUNCTIONS}

Here, we obtain theoretical predictions for the nearestneighbor functions of stealthy disordered ground states. Nearest-neighbor functions describe the probability of finding the nearest point of a point process in $\mathbb{R}^{d}$ at some given distance from a reference point in space. Such statistical quantities are called "void" or "particle" nearest-neighbor functions if the reference point is an arbitrary point of space or an actual point of the point process, respectively [71]. Our focus here is on the particle nearestneighbor functions.

The particle nearest-neighbor probability density function $H_{P}(r)$ is defined such that $H_{P}(r) d r$ gives the probability that the nearest point to the arbitrarily chosen point lies at a distance between $r$ and $r+d r$ from this chosen point of the point process. The probability that a sphere of radius $r$ centered at a point does not have other points, called the exclusion probability $E_{P}(r)$, is the associated complementary cumulative distribution function and so $E_{P}(r)=1-\int_{0}^{r} H_{P}(x) d x$ and hence $H_{P}(r)=-\partial E_{P} / \partial r$.

The nearest-neighbor functions can be expressed as an infinite series whose terms are integrals over $n$-body correlation functions defined in Sec. II [71,72]. In general, an exact evaluation of this infinite series is not possible because the $g_{n}$ are not known accurately for $n \geq 3$, except for simple cases, such as the Poisson point process. Theoretically, one must either devise approximations or rigorous bounds to estimate nearest-neighbor quantities for general models [71,73]. 

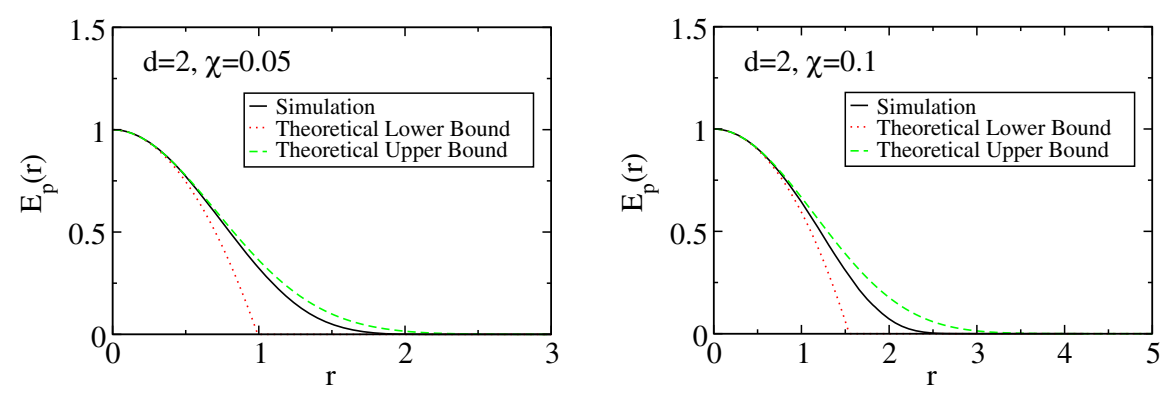

FIG. 10. Comparison of the lower and upper bounds (88) and (89) to our numerical results for the exclusion probability function $E_{P}(r)$ for $\chi=0.05$ and $\chi=0.1$ for $d=2$. Here, $K=1$.
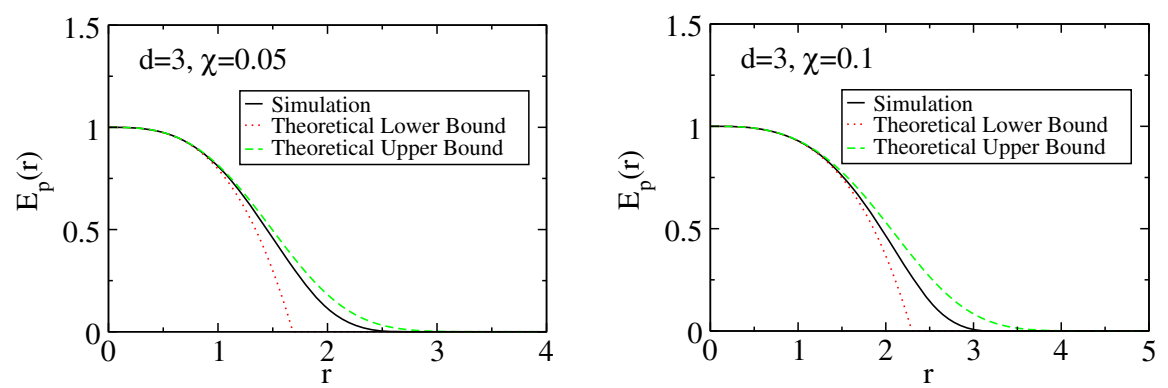

FIG. 11. Comparison of the lower and upper bounds (88) and (89) to our numerical results for the exclusion probability function $E_{P}(r)$ for $\chi=0.05$ and $\chi=0.1$ for $d=3$. Here, $K=1$.

Torquato has given rigorous upper and lower bounds on the so-called canonical $n$-point correlation function $H_{n}$ for point processes in $\mathbb{R}^{d}$ [72,74]. Since nearest-neighbor functions are just special cases of $H_{n}$, then we also have strict bounds on them for such models [71,72]. Here, we employ upper and lower bounds on $E_{P}(r)$, which relies on knowledge of the pair correlation function:

$$
\begin{gathered}
E_{P}(r) \geq 1-Z(r), \\
E_{P}(r) \leq \exp [-Z(r)],
\end{gathered}
$$

where $Z(r)$ is the cumulative coordination number [cf. Eq. (4)]. The upper bound (89) was presented in Ref. [47].

These bounds are evaluated for stealthy ground-state configurations using the analytical expression for the pair correlation function given in Sec. VII. Figures 10 and 11 compare these bounds to our numerical results for both $\chi=0.05$ and $\chi=0.1$ for $d=2$ and $d=3$, respectively. We see that the bounds on $E_{P}(r)$ provide the correct qualitative trends as a function of $r$, the upper bound being the sharper of the two bounds for these cases.

The mean nearest-neighbor distance $\lambda$ is defined as the first moment of $H_{P}(r)$ or, equivalently, zeroth moment of $E_{P}(r)$, i.e.,

$$
\lambda=\int_{0}^{\infty} r H_{P}(r) d r=\int_{0}^{\infty} E_{P}(r) d r .
$$

For an ideal gas (Poisson point process) at number density $\rho$, the mean nearest neighbor can be explicitly given in any dimension [72]:

$$
\lambda_{\text {Ideal }}=\frac{\Gamma(1+1 / d)}{2\left[\rho v_{1}(1 / 2)\right]^{1 / d}}
$$

Using the upper bound (89) and relation (90), we plot in Fig. 12 upper bounds on the mean nearest-neighbor distance $\lambda$, scaled by the corresponding ideal-gas quantity obtained from Eq. (91), as a function of $\chi$ for the first four space dimensions. For fixed $\chi$, the upper bounds on $\lambda / \lambda_{\text {ideal }}$ decrease as the space dimension increases, as expected, and tends to unity in the large- $d$ limit, consistent with the socalled "decorrelation" principle [47,59].

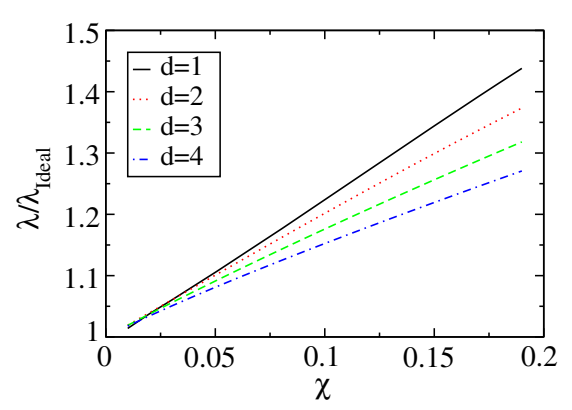

FIG. 12. Upper bounds on the mean nearest-neighbor distance $\lambda$, scaled by $\lambda_{\text {ideal }}$ [cf. Eq. (91)], as a function of $\chi$ for the first four space dimensions. 


\section{EXCITED STATES: STRUCTURE FACTOR AND THERMAL EXPANSION COEFFICIENT}

Here, we derive accurate analytical formulas for the structure factor and thermal expansion coefficient for the excited states associated with stealthy ground states at sufficiently small temperatures. We see from the compressibility relation (3) that if the isothermal compressibility $\kappa_{T}$ is bounded, then $S(0)$ must be zero for any ground state, stealthy or not. Recall that for stealthy ground states, $\kappa_{T}$ is bounded according to Eq. (42). Now consider excited states infinitesimally close to the stealthy ground states, i.e., when temperature $T$ is positive and infinitesimally small. Under the highly plausible assumption that the structure of such excited states will be infinitesimally near the ground-state configurations for sufficiently small $\chi$ and $T$, then to an excellent approximation, the pressure is given by

$$
p \sim \rho T+\frac{\rho^{2}}{2}
$$

where the first term is the ideal-gas contribution and the second term is the configurational contribution, which, under the stated conditions, is effectively the same as the ground-state expression (41), where we have set $k_{B}=v_{0}=K=1$. Thus, relation (92) yields the isothermal compressibility $\kappa_{T}=[\rho(\rho+T)]^{-1}$, which, when substituted into Eq. (3) for large $\rho$ (small $\chi$ ) and small $T$, yields that $S(0)$ varies linearly with $T$ for such excited states:

$$
S(0) \sim C(d) \chi T,
$$

in units $k_{B}=v_{0}=K=1$, where $C(d)=2 d(2 \pi)^{d} / v_{1}(1)$ is a $d$-dependent constant.

Figure 13 shows that the prediction of relation (93) is in excellent agreement with our MD simulation results (Appendix B) in the case $d=2$. It is expected that this positive value of $S(0)$ will be the uniform value of $S(k)$ for $0 \leq k \leq K$ for the special case of the step-function powerlaw potential $\tilde{v}(K)$ [the case $m=0$ in Eq. (15)] for small $\chi$.

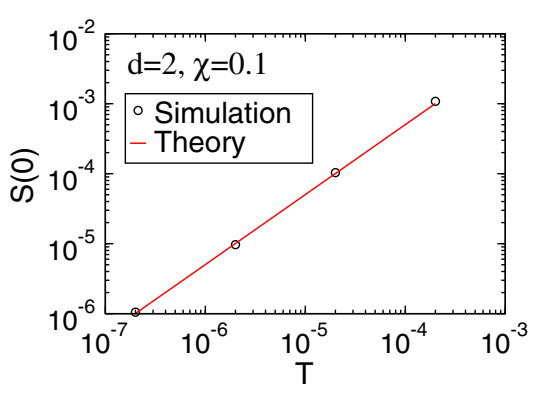

FIG. 13. Comparison of the theoretically predicted structure factor at the origin $S(0)$ versus absolute temperature $T$, as obtained from Eq. (93), to our corresponding simulation results for a two-dimensional stealthy system at $\chi=0.1$. Here, we take $k_{B}=v_{0}=K=1$.
This behavior of $S(k)$ has indeed been verified by our simulation results in various dimensions. For other stealthypotential function choices, $S(k)$ will no longer be a constant for $0 \leq k \leq K$.

An interesting conclusion to be drawn from this analysis is that, for a system, $S(0)$ can be arbitrarily close to zero at positive temperatures, even if $T$ is itself arbitrarily small. This means that, for all practical purposes, such systems at positive $T$ are effectively hyperuniform. Perfect hyperuniformity is not necessarily required in order to achieve novel physical properties in technological applications.

Using the cyclic identity $(\partial \rho / \partial T)_{p}(\partial T / \partial p)_{\rho}(\partial p / \partial \rho)_{T}=$ -1 and approximation (92), it immediately follows that the thermal expansion coefficient $\alpha \equiv-\rho^{-1}(\partial \rho / \partial T)_{p}$, for sufficiently small $\chi$ and $T$, is given by

$$
\alpha \sim C^{2}(d) \chi^{2} T .
$$

We see that the thermal expansion is positive under such conditions, which is to be contrasted with the anomalous negative thermal expansion behavior for sufficiently large $\chi$ over a low temperature range demonstrated in our earlier numerical work $[15,16]$.

\section{CONCLUSIONS AND DISCUSSION}

Stealthy hyperuniform disordered ground states in $\mathbb{R}^{d}$ are infinitely degenerate and arise from a class of bounded longranged pair potentials with compactly supported Fourier transforms. Such exotic many-particle states of matter were previously studied only numerically. Because the configurational dimensionality depends on the density (or $\chi$ ), a highly unusual situation, and there are an infinite number of distinct ways to sample the ground-state manifold, each with its own probability measure, it has been theoretically very challenging to devise predictive ensemble theories. A new type of statistical-mechanical theory needed to be invented. This paper has initiated such a theoretical program.

Specifically, we have derived general exact relations for the ground-state energy, pressure, and isothermal compressibility that apply to any ensemble as a function of the number density $\rho$ in any dimension $d$. We demonstrated how disordered degenerate ground states can arise as part of the ground-state manifold. We also obtained exact integral conditions that both the pair correlation function $g_{2}(r)$ and structure factor $S(k)$ must satisfy in any ensemble. Then, we specialized our results to the canonical ensemble in the zero-temperature limit by exploiting an ansatz that stealthy states behave like pseudoequilibrium hard-sphere systems in Fourier space [75]. The resulting theoretical predictions for $g_{2}(r)$ and $S(k)$ were shown to be in excellent agreement with computer simulations across the first three space dimensions for sufficiently small $\chi$. These results were used to theoretically obtain order metrics, local number variance, and nearest-neighbor functions across dimensions. We also derived accurate analytical formulas for the structure factor and thermal expansion 
coefficient for the excited states associated with stealthy ground states at sufficiently small temperatures. Our analyses provide new insights on our fundamental understanding of the nature and formation of low-temperature states of amorphous matter. Our work also offers challenges to experimentalists to synthesize stealthy ground states at the molecular level, perhaps with polymers, as suggested in Sec. III.

There are many remaining open theoretical problems. While the pseudo-hard-sphere system picture for the canonical ensemble is almost surely exact in the limit $\chi \rightarrow 0$, a future challenge would be to provide rigorous justification for this picture for positive but small $\chi$. One possible avenue that could be pursued is the formulation of an exact perturbation theory for the pair statistics about the weakly constrained limit $(\chi \rightarrow 0)$. As noted in the Introduction, while the configuration space is fully connected for sufficiently small $\chi$, quantifying its topology as a function of $\chi$ up to $\chi_{\max }^{*}$ is an outstanding open problem. At some intermediate range of $\chi$, the topology of the ground-state manifold undergoes a sequence of one or more disconnection events, but this process is poorly understood and demands future study. In the limit $\chi \rightarrow \chi_{\max }^{*}$, the disconnection becomes complete at the unique crystal ground state [77].

The simple constraint and degrees-of-freedom counting arguments described earlier lead to definite predictions for the entropically favored stealthy ground states derived from the canonical ensemble in the limit $T \rightarrow 0$. For $\chi$ between 0 and $1 / 2$, the ground states are disordered and possess a configurational dimension per particle $d_{C}$ of $d(1-2 \chi)$; see Eq. (37). At $\chi=1 / 2$, the configurational dimensionality per particle collapses to zero, and there is a concomitant phase transition to a crystal phase. The fact that the pressure is a continuous function of density [cf. Eq. (41)] for all $\chi$ up to $\chi_{\max }^{*}$ (see Tables I-IV) implies that any phase transition could be continuous. While this eliminates a first-order phase transition in which the phase densities are unequal, it does not prohibit such a phase transition in which the two distinct phases possess the same number density. At $\chi=0.5$, our numerical evidence indicates that the structurally distinct fluid and crystal phases have equal free energies. This does not necessarily imply that the two phases could coexist side by side within the system separated by an interface. This phase diagram is depicted in Fig. 14, which applies to the first four space dimensions. For $d=1$, the only crystal phase allowed is the integer lattice (see Sec. VI), and hence there can be no phase coexistence. For $d=2$, our simulations [14-16,76] indicate that the crystal phase is the triangular lattice for $1 / 2 \leq \chi \leq \chi_{\max }^{*}$. However, for $d=3$, it is possible that there may be more than one crystal phase. For example, while for sufficiently high $\chi$ up to $\chi_{\max }^{*}$, we expect the stable crystal to be the BCC lattice, our simulations cannot eliminate the possibility that the FCC lattice is a stable phase for some $\chi$ in the range $1 / 2 \leq \chi \leq 0.9068 \ldots$; for $\chi>0.9068 \ldots$, the FCC lattice cannot be a ground state

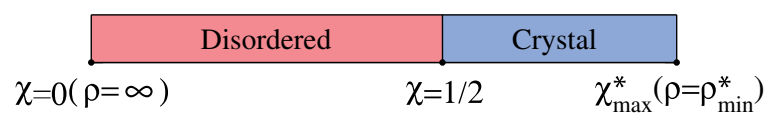

FIG. 14. Phase diagram for the entropically favored stealthy ground states in the canonical ensemble as a function of $\chi$ which applies to the first four space dimensions.

(see Table III). Since four dimensions is more similar to two dimensions in that the lattice corresponding to $\chi_{\max }^{*}$ is equivalent to its dual, we would expect that the $D_{4}$ lattice is the stable crystal for $1 / 2 \leq \chi \leq \chi_{\max }^{*}$, but this remains to be confirmed.

All of our previous and current simulations for the first three space dimensions [12-16] strongly suggest that all of the energy minima attained were global ones for $\chi<0.5$, but when $\chi>0.5$, the topography of the energy landscape suddenly exhibits local minima above the ground-state energies. The possible configurations that can arise as part of the ground-state manifold for $\chi>1 / 2$, regardless of their probability of occurrence, not only include periodic crystals for $d \geq 2$, as discussed in Sec. VI, but also aperiodic structures, reflecting the complex nature of the energy landscape. For example, for $d=2$, the manifold includes generally aperiodic "wavy" phases, which have been shown to arise via numerical energy minimizations from random initial conditions with high probability in a range of $\chi$ where the triangular lattice is entropically favored $[12,14]$. A deeper understanding of such aspects of the ground-state manifold would undoubtedly shed light on the topography of the energy landscape.

\section{ACKNOWLEDGMENTS}

This research was supported by the U.S. Department of Energy, Office of Basic Energy Sciences, Division of Materials Sciences and Engineering, under Grant No. DEFG02-04-ER46108.

\section{APPENDIX A: COMMON $d$-DIMENSIONAL LATTICES}

Common $d$-dimensional lattices include the hypercubic $\mathbb{Z}^{d}$, checkerboard $D_{d}$, and root $A_{d}$ lattices, defined, respectively, by

$$
\begin{gathered}
\mathbb{Z}^{d}=\left\{\left(x_{1}, \ldots, x_{d}\right): x_{i} \in \mathbb{Z}\right\} \quad \text { for } d \geq 1, \quad(\mathrm{~A} 1) \\
D_{d}=\left\{\left(x_{1}, \ldots, x_{d}\right) \in \mathbb{Z}^{d}: x_{1}+\cdots+x_{d} \text { even }\right\} \quad \text { for } d \geq 3,
\end{gathered}
$$

$$
\begin{aligned}
& A_{d}=\left\{\left(x_{0}, x_{1}, \ldots, x_{d}\right) \in \mathbb{Z}^{d+1}: x_{0}+x_{1}+\cdots+x_{d}=0\right\} \\
& \quad \text { for } d \geq 1
\end{aligned}
$$

where $\mathbb{Z}$ is the set of integers $(\ldots-3,-2,-1,0,1,2,3 \ldots)$; $x_{1}, \ldots, x_{d}$ denote the components of a lattice vector of either 
$\mathbb{Z}^{d}$ or $D_{d}$; and $x_{0}, x_{1}, \ldots, x_{d}$ denote a lattice vector of $A_{d}$. The $d$-dimensional lattices $\mathbb{Z}_{*}^{d}, D_{d}^{*}$, and $A_{d}^{*}$ are the corresponding dual lattices. Following Conway and Sloane [78], we say that two lattices are equivalent or similar if one becomes identical to the other possibly by a rotation, reflection, and change of scale, for which we use the symbol $\equiv$. The $A_{d}$ and $D_{d}$ lattices can be regarded as $d$ dimensional generalizations of the face-centered-cubic (FCC) lattice defined by $A_{3} \equiv D_{3}$; however, for $d \geq 4$, they are no longer equivalent. In two dimensions, $A_{2} \equiv A_{2}^{*}$ defines the triangular lattice with a dual lattice that is equivalent. In three dimensions, $A_{3}^{*} \equiv D_{3}^{*}$ defines the bodycentered-cubic (BCC) lattice. In four dimensions, the checkerboard lattice and its dual are equivalent, i.e., $D_{4} \equiv D_{4}^{*}$. The hypercubic lattice $\mathbb{Z}^{d} \equiv \mathbb{Z}_{*}^{d}$ and its dual lattice are equivalent for all $d$.

\section{APPENDIX B: SIMULATION PROCEDURE TO GENERATE AND SAMPLE STEALTHY GROUND STATES IN THE CANONICAL ENSEMBLE}

To numerically sample the stealthy ground-state manifold in the disordered regime in the canonical ensemble in the $T \rightarrow 0$ limit for $d=1,2$, and 3 , we performed molecular dynamics (MD) simulations at a very low dimensionless equilibration temperature $T_{E} \equiv k_{B} T /\left(v_{0} K^{d}\right)$, periodically took configurational "snapshots," and then used these configurations as input to the L-BFGS optimization algorithm [79] to get the corresponding ground states. The dimensionless temperatures that we use are $T_{E}=2 \times 10^{-4}$ for $d=1, T_{E}=$ $2 \times 10^{-6}$ for $d=2$, and $T_{E}=1 \times 10^{-6}$ for $d=3$. The equilibration temperature at a fixed dimension was chosen so that no changes in the pair correlation function are observed over some range of equilibration temperatures. The MD simulations were first performed in the microcanonical ensemble using the velocity Verlet algorithm [80]. The time steps were chosen so that the relative energy change every 3000 time steps is less than $10^{-8}$. However, to enforce the desired temperature, we also performed MD simulations in the canonical ensemble using an Anderson thermostat [80]. We employed fifteen million time steps to equilibrate a system. After that, a snapshot was taken every 3000 time steps for further energy minimization. Because stealthy potentials in direct space are long-ranged, the energy is most accurately calculated in Fourier space using Eq. (1). The numerical errors in the achieved groundstate energies are extremely small, usually on the order of $10^{-20}$ (in units of $v_{0} K^{d}$ ). The force on the $j$ th particle is calculated using the gradient of Eq. (1), yielding $\mathbf{F}_{j}=$ $-\nabla_{j} \Psi\left(\mathbf{r}^{N}\right)=\left(1 / v_{F}\right) \sum_{\mathbf{k}} \mathbf{k} \tilde{v}(\mathbf{k}) \operatorname{Im}\left[\tilde{n}(\mathbf{k}) \exp \left(i \mathbf{k} \cdot \mathbf{r}_{j}\right)\right]$. The number of particles in the simulation box, $N$, is calculated from Eq. (2) for given $d, \chi$, and $M(K)$. We chose $M(K)=$ 50 for $d=1, M(K)=54$ for $d=2$, and $M(K)=39$ for $d=3$. The fundamental cell employed is the one corresponding to the crystal with the largest value of $\chi_{\max }$ in each dimension (see Tables I-IV).

Structural characteristics, such as the pair correlation function $g_{2}(r)$, structure factor $S(k)$, number variance $\sigma^{2}(R)$, and nearest-neighbor function $E_{P}(r)$, are obtained by sampling each generated configuration for a fixed value of $\chi$ and ensemble averaging over at least 20000 configurations. Two power-law potentials (15) were used: one with $m=0$ and the other with $m=2$. As expected, both potentials produced the same ensemble-averaged structural properties to within small numerical errors (as explained in Sec. IV B), the agreement of which provides a good test on the validity of the simulation results. Our simulation results for $g_{2}(r)$ and $S(k)$ also satisfied the exact integral conditions presented in Secs. VA and V B. Additional simulation details will be described elsewhere [76].

[1] F.H. Stillinger, Phase transitions in the Gaussian core system, J. Chem. Phys. 65, 3968 (1976).

[2] D. Frenkel, B. M. Mulder, and J. P. McTague, Phase Diagram of a System of Hard Ellipsoids, Phys. Rev. Lett. 52, 287 (1984).

[3] P. M. Chaikin and T. C. Lubensky, Principles of Condensed Matter Physics (Cambridge University Press, New York, 1995).

[4] M. Guenza and K. S. Schweizer, Local and microdomain concentration fluctuation effects in block copolymer solutions, Macromolecules 30, 4205 (1997).

[5] J. C. Crocker, M. T. Valentine, E. R. Weeks, T. Gisler, P. D. Kaplan, A. G. Yodh, and D. A. Weitz, Two-Point Microrheology of Inhomogeneous Soft Materials, Phys. Rev. Lett. 85, 888 (2000).

[6] M. P. Valignat, O. Theodoly, J. C. Crocker, W. B. Russel, and P. M. Chaikin, Reversible self-assembly and directed assembly of DNA-linked micrometer-sized colloids, Proc. Natl. Acad. Sci. U.S.A. 102, 4225 (2005).

[7] B. M. Mladek, D. Gottwald, G. Kahl, M. Neumann, and C. N. Likos, Formation of Polymorphic Cluster Phases for a Class of Models of Purely Repulsive Soft Spheres, Phys. Rev. Lett. 96, 045701 (2006).

[8] C. E. Zachary, F. H. Stillinger, and S. Torquato, Gaussiancore model phase diagram and pair correlations in high Euclidean dimensions, J. Chem. Phys. 128, 224505 (2008).

[9] B. Capone, I. Coluzza, F. LoVerso, C. N. Likos, and R. Blaak, Telechelic Star Polymers as Self-Assembling Units from the Molecular to the Macroscopic Scale, Phys. Rev. Lett. 109, 238301 (2012).

[10] C. Hanel, C. N. Likos, and R. Blaak, Effective interactions between multilayered ionic microgels, Materials 7, 7689 (2014).

[11] Y. Fan, J. K. Percus, D. K. Stillinger, and F. H. Stillinger, Constraints on collective density variables: One dimension, Phys. Rev. A 44, 2394 (1991).

[12] O. U. Uche, F. H. Stillinger, and S. Torquato, Constraints on collective density variables: Two dimensions, Phys. Rev. E 70, 046122 (2004). 
[13] O. U. Uche, S. Torquato, and F. H. Stillinger, Collective coordinates control of density distributions, Phys. Rev. E 74, 031104 (2006).

[14] R. D. Batten, F. H. Stillinger, and S. Torquato, Classical disordered ground states: Super-ideal gases, and stealth and equi-luminous materials, J. Appl. Phys. 104, 033504 (2008).

[15] R. D. Batten, F. H. Stillinger, and S. Torquato, Novel LowTemperature Behavior in Classical Many-Particle Systems, Phys. Rev. Lett. 103, 050602 (2009).

[16] R. D. Batten, F. H. Stillinger, and S. Torquato, Interactions leading to disordered ground states and unusual lowtemperature behavior, Phys. Rev. E 80, 031105 (2009).

[17] C. E. Zachary and S. Torquato, Anomalous local coordination, density fluctuations, and void statistics in disordered hyperuniform many-particle ground states, Phys. Rev. E 83, 051133 (2011).

[18] S. Martis, É. Marcotte, F. H. Stillinger, and S. Torquato, Exotic ground states of directional pair potentials via collective-density variables, J. Stat. Phys. 150, 414 (2013).

[19] A. Sütő, Crystalline Ground States for Classical Particles, Phys. Rev. Lett. 95, 265501 (2005).

[20] Indeed, the number of ground-state degeneracies is uncountably infinite for a finite number of particles, which distinguishes it from disordered ground states found in classical Ising-like spin systems in which the number of degeneracies is finite for a finite number of spins [21-24]. We note in passing that while quantum spin liquids have disordered ground states, they are effectively unique [21].

[21] L. Balents, Spin liquids in frustrated magnets, Nature (London) 464, 199 (2010).

[22] C. L. Henley, The "Coulomb Phase" in frustrated systems, Annu. Rev. Condens. Matter Phys. 1, 179 (2010).

[23] R. A. DiStasio, É. Marcotte, R. Car, F. H. Stillinger, and S. Torquato, Designer spin systems via inverse statistical mechanics, Phys. Rev. B 88, 134104 (2013).

[24] É. Marcotte, R. A. DiStasio, F. H. Stillinger, and S. Torquato, Designer spin systems via inverse statistical mechanics II. Ground-state enumeration and classification, Phys. Rev. B 88, 184432 (2013).

[25] M. Florescu, S. Torquato, and P. J. Steinhardt, Designer disordered materials with large complete photonic band gaps, Proc. Natl. Acad. Sci. U.S.A. 106, 20658 (2009).

[26] M. Florescu, P. J. Steinhardt, and S. Torquato, Optical cavities and waveguides in hyperuniform disordered photonic solids, Phys. Rev. B 87, 165116 (2013).

[27] W. Man, M. Florescu, E. P. Williamson, Y. He, S. R. Hashemizad, B. Y. C. Leung, D. R. Liner, S. Torquato, P. M. Chaikin, and P. J. Steinhardt, Isotropic band gaps and freeform waveguides observed in hyperuniform disordered photonic solids, Proc. Natl. Acad. Sci. U.S.A. 110, 15886 (2013).

[28] More generally, stealthy configurations can be those ground states that correspond to minimizing $S(k)$ to be zero at other sets of wave vectors, not necessarily in a connected set around the origin, specific examples of which were investigated in Ref. [14]. We have also used the collectivecoordinate technique to target more general forms of the structure factor for a prescribed set of wave vectors such that $S(\mathbf{k})$ is not minimized to be zero in this set (e.g., powerlaw forms and positive constants) [13,14,17]. There, the resulting configurations are the ground states of interacting many-particle systems with 2-, 3-, and 4-body interactions.

[29] Since both the real and imaginary contributions to $|\tilde{n}(\mathbf{k})|$ are zero for each wave vector $\mathbf{k}$ in the constrained region or exclusion zone, the total number of independent constrained degrees of freedom is $2 M(K)$. Hence, in the large-system limit, $\chi=1 / 2$ is the critical value when there are no longer any degrees of freedom that can be independently constrained to be zero (not $\chi=1$ ), according to this simple counting argument. The reason why we use the definition (2) is that in the more general case when $|\tilde{n}(\mathbf{k})|$ is constrained to be positive (not zero) for some set of wave vectors $[13,14,17], \chi=1$ is indeed the critical value when one runs out of degrees of freedom that can be independently constrained in the large-system limit.

[30] The limit $\rho \rightarrow \infty$ described in this caption does not commute with the limit of the number density $\rho$ going to infinity, where $\rho$ is derived from the thermodynamic limit defined in Eq. (30), which is the situation that will be employed throughout the remainder of the paper. If we take the thermodynamic limit first and then take the limit $\rho \rightarrow \infty$, the pair correlation function tends to the trivial ideal-gas form, but the structure factor does not, as detailed in Sec. VII A.

[31] S. Torquato and F. H. Stillinger, Local density fluctuations, hyperuniform systems, and order metrics, Phys. Rev. E 68 , 041113 (2003).

[32] C. E. Zachary and S. Torquato, Hyperuniformity in point patterns and two-phase heterogeneous media, J. Stat. Mech. (2009) P12015.

[33] A. Donev, F. H. Stillinger, and S. Torquato, Unexpected Density Fluctuations in Disordered Jammed Hard-Sphere Packings, Phys. Rev. Lett. 95, 090604 (2005).

[34] C. E. Zachary, Y. Jiao, and S. Torquato, Hyperuniform Long-Range Correlations Are a Signature of Disordered Jammed Hard-Particle Packings, Phys. Rev. Lett. 106, 178001 (2011).

[35] Y. Jiao and S. Torquato, Maximally random jammed packings of platonic solids: Hyperuniform long-range correlations and isostaticity, Phys. Rev. E 84, 041309 (2011).

[36] D. Chen, Y. Jiao, and S. Torquato, Equilibrium phase behavior and maximally random jammed state of truncated tetrahedra, J. Phys. Chem. B 118, 7981 (2014).

[37] L. Berthier, P. Chaudhuri, C. Coulais, O. Dauchot, and P. Sollich, Suppressed Compressibility at Large Scale in Jammed Packings of Size-Disperse Spheres, Phys. Rev. Lett. 106, 120601 (2011).

[38] R. Kurita and E. R. Weeks, Incompressibility of polydisperse random-close-packed colloidal particles, Phys. Rev. E 84, 030401 (2011).

[39] R. Dreyfus, Y. Xu, T. Still, L. A. Hough, A. G. Yodh, and S. Torquato, Diagnosing hyperuniformity in two-dimensional, disordered, jammed packings of soft spheres, Phys. Rev. E 91, 012302 (2015).

[40] I. Lesanovsky and J. P. Garrahan, Out-of-equilibrium structures in strongly interacting Rydberg gases with dissipation, Phys. Rev. A 90, 011603 (2014). 
[41] D. Hexner and D. Levine, Hyperuniformity of Critical Absorbing States, Phys. Rev. Lett. 114, 110602 (2015).

[42] R. L. Jack, I. R. Thompson, and P. Sollich, Hyperuniformity and Phase Separation in Biased Ensembles of Trajectories for Diffusive Systems, Phys. Rev. Lett. 114, 060601 (2015).

[43] C. De Rosa, F. Auriemma, C. Diletto, R. Di Girolamo, A. Malafronte, P. Morvillo, G. Zito, G. Rusciano, G. Pesce, and A. Sasso, Toward hyperuniform disordered plasmonic nanostructures for reproducible surface-enhanced Raman spectroscopy, Phys. Chem. Chem. Phys. 17, 8061 (2015).

[44] R. Degl'Innocenti, Y. D. Shah, L. Masini, A. Ronzani, A. Pitanti, Y. Ren, D. S. Jessop, A. Tredicucci, H. E. Beere, and D. A. Ritchie, $\mathrm{THz}$ quantum cascade lasers based on a hyperuniform design, Proc. SPIE Int. Soc. Opt. Eng. 9370, 93700A-1 (2015).

[45] S. Yu, X. Piao, J. Hong, and N. Park, Bloch-Like Wave Dynamics in Disordered Potentials Based on Supersymmetry, arXiv:1501.02591.

[46] Y. Jiao, T. Lau, H. Hatzikirou, M. Meyer-Hermann, J. C. Corbo, and S. Torquato, Avian photoreceptor patterns represent a disordered hyperuniform solution to a multiscale packing problem, Phys. Rev. E 89, 022721 (2014).

[47] S. Torquato, A. Scardicchio, and C. E. Zachary, Point processes in arbitrary dimension from Fermionic gases, random matrix theory, and number theory, J. Stat. Mech. (2008) P11019.

[48] J. Haberko, N. Muller, and F. Scheffold, Direct laser writing of three dimensional network structures as templates for disordered photonic materials, Phys. Rev. A 88, 043822 (2013).

[49] P. Laurin, M. Girard, A. Markov, and M. Skorobogatiy, Hollow Core Terahertz Optical Fibers with Hyperuniform Disordered Dielectric Reflectors, in Proceedings of the 39th International Conference on Infrared, Millimeter, and Terahertz Waves (IRMMW-THz) (2014), pp. 1-2.

[50] M. Hejna, P. J. Steinhardt, and S. Torquato, Nearly hyperuniform network models of amorphous silicon, Phys. Rev. B 87, 245204 (2013).

[51] R. Xie, G. G. Long, S. J. Weigand, S. C. Moss, T. Carvalho, S. Roorda, M. Hejna, S. Torquato, and P. J Steinhardt, Hyperuniformity in amorphous silicon based on the measurement of the infinite-wavelength limit of the structure factor, Proc. Natl. Acad. Sci. U.S.A. 110, 13250 (2013).

[52] R. Ben Brahim and A. Chehaidar, Small-angle X-ray scattering of two-phase atomistic models for amorphous SiliconGermanium alloys, J. Non-Cryst. Solids 416, 4 (2015).

[53] R. B. Batten, F. H. Stillinger, and S. Torquato, Inherent structures for a soft long-range interaction in two-dimensional many-particle systems, J. Chem. Phys. 135, 054104 (2011).

[54] J. P. Hansen and I. R. McDonald, Theory of Simple Liquids (Academic Press, New York, 1986).

[55] The number density $\rho$ can be thought of as arising from a limiting process in which $N$ points are contained within a large region of volume $V$ in $\mathbb{R}^{d}$ such that $\rho \equiv \lim _{N \rightarrow \infty, V \rightarrow \infty} N / V$, the so-called thermodynamic limit.

[56] N. W. Ashcroft and D. N. Mermin, Solid State Physics (Thomson Learning, Toronto, 1976).
[57] Á. Bácsi and A. Virosztek, Local density of states and Friedel oscillations in graphene, Phys. Rev. B 82, 193405 (2010).

[58] M. Abramowitz and I. A. Stegun, Handbook of Mathematical Functions (Dover, New York, 1972).

[59] S. Torquato and F. H. Stillinger, New conjectural lower bounds on the optimal density of sphere packings, Exp. Math. 15, 307 (2006).

[60] Alternatively, for fixed $\rho$ and $d, \chi$ is proportional to $K^{d}$, and hence as $\chi$ is varied, the stealthy potential accordingly changes. This scenario has been considered in some previous numerical work $[12,13]$.

[61] This relation immediately follows from the fact that there are $d N$ degrees of freedom and $2 M$ constraints.

[62] In Ref. [14], it was shown numerically that pressure computed via the viral route for $d=2$ diverged for the step-function stealthy potential but converged for the stealthy overlap potential.

[63] C. E. Zachary and S. Torquato, High-dimensional generalizations of the Kagome and diamond crystals and the decorrelation principle for periodic sphere packings, J. Stat. Mech. (2011) P10017.

[64] J. H. Conway and N. J. A. Sloane, On lattices equivalent to their duals, J. Number Theory 48, 373 (1994).

[65] Particle permutations between geometrically equivalent but disconnected configurations are not regarded as degeneracies.

[66] The constant $\alpha(k=K ; K)$ is trivially related to the third virial coefficient of hard hyperspheres and has a connection to continuum percolation of overlapping hyperspheres $[54,67]$.

[67] S. Torquato, Effect of dimensionality on the continuum percolation of overlapping hyperspheres and hypercubes, J. Chem. Phys. 136, 054106 (2012).

[68] This positive dimensionless order metric is similar in spirit to those involving one-dimensional integrals of either absolute values of $h(r)$ [69] or $h^{2}(r)$ [70].

[69] T. M. Truskett, S. Torquato, and P. G. Debenedetti, Towards a quantification of disorder in materials: Distinguishing equilibrium and glassy sphere packings, Phys. Rev. E 62, 993 (2000).

[70] A. Andreanov, A. Scardicchio, and S. Torquato, Extreme Lattices: Symmetries and Decorrelations, arXiv:1309.1301.

[71] S. Torquato, B. Lu, and J. Rubinstein, Nearest-neighbor distribution functions in many-body systems, Phys. Rev. A 41, 2059 (1990).

[72] S. Torquato, Random Heterogeneous Materials: Microstructure and Macroscopic Properties (Springer-Verlag, New York, 2002).

[73] S. Torquato, Mean Nearest-Neighbor Distance in Random Packings of Hard d-Dimensional Spheres, Phys. Rev. Lett. 74, 2156 (1995).

[74] S. Torquato, Microstructure characterization and bulk properties of disordered two-phase media, J. Stat. Phys. 45, 843 (1986).

[75] It is interesting to note that classical hard-sphere configurations in direct space can be viewed as degenerate ground states, but trivially so. Importantly, while the probability measure of the hard-sphere system is a constant over its entire ground-state manifold, that of the stealthy ground 
states is not a constant; see Ref. [76] for further details on this point.

[76] G. Zhang, F. H. Stillinger, and S. Torquato, unpublished.

[77] For a finite system of $N$ particles, the ground-state manifold when $\chi=\chi_{\max }^{*}$ consists of $(N-1)$ ! permutationally equivalent configurations that cannot be connected by continuous configurational paths in the manifold.
[78] J. H. Conway and N. J. A. Sloane, Sphere Packings, Lattices and Groups (Springer-Verlag, New York, 1998).

[79] D. C. Liu and J. Nocedal, On the limited memory BFGS method for large scale optimization, Math. Program. 45, 503 (1989).

[80] D. Frenkel and B. Smit, Understanding Molecular Simulation (Academic Press, New York, 1996). 